\title{
Time-Resolved Investigations of Electronic Transport Dynamics in Quantum Cascade Lasers Based on Diagonal Lasing Transition
}

\section{Citation}

Choi, Hyunyong, Laurent Diehl, Zong-Kwei Wu, Marcella Giovannini, Jerome Faist, Federico Capasso, Theodore B. Norris. 2009. Time-resolved investigations of electronic transport dynamics in quantum cascade lasers based on diagonal lasing transition. IEEE Journal of Quantum Electronics 45(4): 307-321.

\section{Published Version}

doi:10.1109/JQE.2009.2013091

\section{Permanent link}

http://nrs.harvard.edu/urn-3:HUL.InstRepos:3445985

\section{Terms of Use}

This article was downloaded from Harvard University's DASH repository, and is made available under the terms and conditions applicable to Other Posted Material, as set forth at http:// nrs.harvard.edu/urn-3:HUL.InstRepos:dash.current.terms-of-use\#LAA

\section{Share Your Story}

The Harvard community has made this article openly available.

Please share how this access benefits you. Submit a story.

Accessibility 


\title{
Time-Resolved Investigations of Electronic Transport Dynamics in Quantum Cascade Lasers Based on Diagonal Lasing Transition
}

\author{
Hyunyong Choi, Member, IEEE, Laurent Diehl, Zong-Kwei Wu, Marcella Giovannini, Member, IEEE,
} Jérôme Faist, Member, IEEE, Federico Capasso, Fellow, IEEE, and Theodore B. Norris, Associate Member, IEEE

\begin{abstract}
In this study, the nature of electronic transport in quantum cascade lasers (QCLs) has been extensively investigated using an ultrafast time-resolved, degenerate, pump-probe optical technique. Our investigations enable a comprehensive understanding of the gain recovery dynamics in terms of a coupling of the electronic transport to the oscillating intracavity laser intensity. In QCLs that have a lasing transition diagonal in real space, studies of the near-threshold reveal that the transport of electrons changes bias region from phonon-limited relaxation (tens of picoseconds) below threshold to photon-driven transport via stimulated emission (a few picoseconds) above threshold. The gain recovery dynamics in the photon-driven regime is compared with conventional four-level lasers such as atomic, molecular, and semiconductor interband lasers. The depopulation dynamics out of the lower lasing state is explained using a tight-binding tunneling model and phonon-limited relaxation. For the superlattice relaxation, it is possible to explain the characteristic picosecond transport via dielectric relaxation; Monte Carlo simulations with a simple resistor model are developed, and the Esaki-Tsu model is applied. Subpicosecond dynamics due to carrier heating in the upper subband are isolated and appear to be at most about $10 \%$ of the gain compression compared with the contribution of stimulated emission. Finally, the polarization anisotropy in the active waveguide is experimentally shown to be negligible on our pump-probe data, supporting our interpretation of data in terms of gain recovery and transport.
\end{abstract}

Manuscript received May 19, 2008; revised August 21, 2008 and October 16, 2008. Current version published March 11, 2009. This work was supported by the U.S. Army Research Office.

H. Choi was with the Center for Ultrafast Optical Science, Department of Electrical Engineering and Computer Science, University of Michigan, Ann Arbor, MI 48109-2099 USA. He is now with the Materials Sciences Division, Lawrence Berkeley National Laboratory and Department of Physics, University of California, Berkeley, CA 94720 USA (e-mail: hychoi@lbl.gov; hychoi@berkeley.edu).

L. Diehl and F. Capasso are with the School of Engineering and Applied Sciences, Harvard University, Cambridge, MA 02138 USA (e-mail: 1diehl@ seas. harvard.edu; capasso@deas.harvard.edu).

Z.-K. Wu was with the Center for Ultrafast Optical Science, Department of Electrical Engineering and Computer Science, University of Michigan, Ann Arbor, MI 48109-2099 USA. He is now with Intel Corporation, Santa Clara, CA 95054 USA (e-mail: zjw @eecs.umich.edu).

M. Giovannini is with the Institute of Physics, University of Neuchâtel, CH-2000 Neuchâtel, Switzerland.

J. Faist was with the Institute of Physics, University of Neuchâtel, CH-2000 Neuchâtel, Switzlerand. He is now with ETH Zurich, CH-8092 Zurich, Switzerland (e-mail: jerome.faist@ phys.ethz.ch).

T. B. Norris is with the Center for Ultrafast Optical Science, Department of Electrical Engineering and Computer Science, University of Michigan, Ann Arbor, MI 48109-2099 USA (e-mail: tnorris@eecs.umich.edu).

Color versions of one or more of the figures in this paper are available online at http://ieeexplore.ieee.org.

Digital Object Identifier 10.1109/JQE.2009.2013091
Index Terms-Carrier dynamics, electronic transport, midinfrared (mid-IR), quantum cascade laser (QCL), time-resolved.

\section{INTRODUCTION}

$\mathbf{I}$ $\mathrm{N}$ A quantum cascade laser (QCL), electrons cascade through the heterostructure, ideally emitting one photon via stimulated emission in each active region. Thus, QCL operation results from a coupling of perpendicular electronic transport with the intracavity optical field. Since the first demonstration of a QCL in 1994 [1], remarkable advances in device performance have been made. Scientifically and technologically important examples are the development of high-power MBE- and MOVPE-grown QCL operating in CW at room temperature and above [2]-[4], high-speed electrical modulation without relaxation oscillation [5] and observation of Bloch gain in QCLs [6], and the realization of QC structures emitting in the terahertz $(\mathrm{THz})$ range of the spectrum [7], [8]. However, the mechanism of electronic transport across the active and injector regions, which is closely related to the dynamic gain recovery, has not been extensively investigated, though there have been some studies of carrier transport and relaxation processes in nonlasing quantum-well (QW) structures [9]-[12].

Our approach to studying the nature of electronic transport in an operating QCL is to use femtosecond pump-probe spectroscopy to measure the dynamics of gain recovery. Pump-probe methods have previously been used to explore the possibility of coherent transport in resonant-tunneling QC structures [13], [14], which has been the subject of considerable theoretical interest [15]-[17]. The structure used in those experiments, however, was not an operating laser and thus is not directly relevant to the dynamics under working conditions; to date, most information on the electron transport has been obtained only through measurements of steady-state light or voltage versus current characteristics $(L-I-V)$ [18]. One recent pulse-propagation study on an operating THz QCL has measured the $\pi$ phase flip as an effect of the stimulated emission on the propagating pulses [19].

In this study, we explore the gain recovery dynamics of an operating QCL below and above threshold. We observe how the perpendicular transport of electrons across the active region is driven by the laser photons. Following an impulsive perturbation of the intracavity field, the gain recovery is time-resolved and the measured data are used to model the QCL level density 
coupled to the cavity photon density rate equation. The developed rate-equation model is then used to calculate the steadystate light-current-voltage $(L-I-V)$ relationship, thus leading to a self-consistent picture of laser oscillation and transport dynamics. The resulting model highlights the substantial difference in the gain recovery between QCLs and other types of laser, arising from the central role played by transport. Note that this paper presents all of the details of the gain recovery dynamics presented in [20], including a more complete classical and quantum mechanical description of the full electronic transport dynamics.

We also show how several unique features of the QCL will enable us the observation of photon-driven transport. The most important of these is that the gain region in a QCL is an open system (coupled by transport through the injector superlattice), whereas the gain medium in conventional laser systems such as atomic, molecular, and semiconductor interband lasers constitutes a closed system. Thus, the QCL manifests qualitatively different gain recovery and transport physics.

In addition to the photon-driven transport across the active region, we also present detailed analysis of the lower lasing state emptying and superlattice transport dynamics. For the lower lasing state, we describe the transport in terms of the densitymatrix tunneling model and phonon-assisted scattering process. For the superlattice transport, we present a simple, but self-consistent dielectric relaxation model to understand the contribution of the perpendicular electron transport across the superlattice injector to the gain recovery dynamics.

The organization of this paper is as follows. DC characterization of the QCLs and the time-resolved pump-probe methods are described in Section II. The self-consistent rate-equation model used for data analysis is presented in Section III. Section IV focuses on the photon-driven transport in the active region. The gain dynamics of four-level conventional laser systems is compared with that of QCLs in this section. In Section V, we discuss the depopulation dynamics out of the lower lasing state. Superlattice transport effects are discussed in Section VI. Possible experimental artifacts during the differential transmission (DT) measurements are discussed in Section VII. Finally, Section VIII concludes our discussion.

\section{QCL ChARACTERISTICS AND TIME-RESOlVED DT MEASUREMENTS}

\section{A. QCL Characteristics}

Two QCLs (N432 and N433) are used in the study. Self-consistent conduction bandstructures are shown in Fig. 1. Both are based on so called "diagonal transition," in which the lasing transition is diagonal in real space. The population inversion is approximately constant as a function of bias, and the gain is tuned by varying the wavefunction overlap ("oscillator strength tuning") via the bias voltage [21]. The two lasers have different threshold current, so that the bias dependence of the gain recovery dynamics around the threshold can be investigated.

The two QCLs have somewhat different band structure. Sample N433 differs from N432 mainly in that it incorporates a saturable absorber layer, such as has been used for investigations of multimode behavior, temporal pulsations,

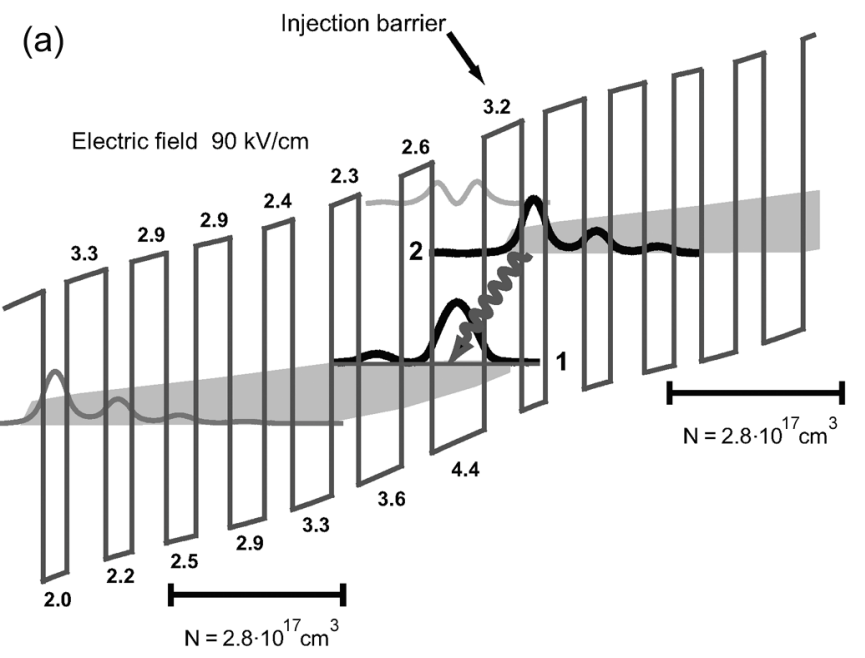

(b)

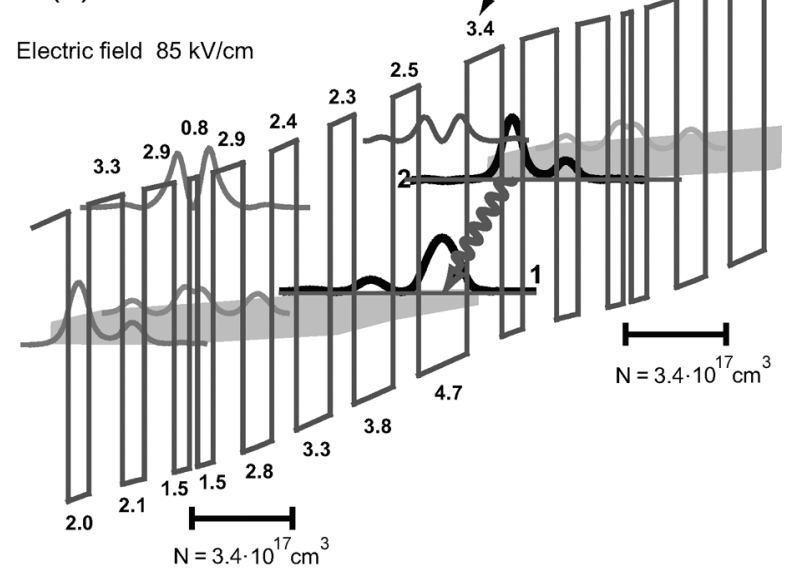

Fig. 1. (a) Self-consistent bandstructure calculation of the $\mathrm{In}_{0.6} \mathrm{Ga}_{0.4} \mathrm{As} / \mathrm{In}_{0.44} \mathrm{Al}_{0.56} \mathrm{As}$ strained layer sequence for two periods of the cascade heterostructure, at a bias corresponding to an applied electric field of $90 \mathrm{kV} / \mathrm{cm}$, is displayed. Layer thicknesses are given in nanometers. The wavefunctions for the upper $(|2\rangle)$ and lower lasing states $(|1\rangle)$ are shown, illustrating the diagonal nature of the transition in real space. The wavy arrow indicates the lasing transition. The miniband is indicated by the shaded gray region. The horizontal segments show the doped region for each period; $N$ is the donor density. (b) Corresponding bandstructure calculation for N433 at a bias corresponding to an applied electric field of $85 \mathrm{kV} / \mathrm{cm}$ is displayed.

and instabilities [22]. For the experiments reported here, the saturable absorber plays no role, since these effects occur only when the laser is biased far above threshold, and our experiments are mainly concentrated on the laser operation below and just above threshold.

This particular QCL design can offer an ideal laboratory to investigate a close coupling of the electronic transport to lasing. As shown in Fig. 1(a) and (b), electrons propagate through the superlattice region, lose their energy, and relax to the injector ground state, but the injector ground state is also the upper lasing state in this structure. When an appropriate bias is applied across the structure, a population inversion can be achieved even under conditions of a small value of the dipole matrix element $z_{21}$ ( $~ 0.3 \mathrm{~nm}$ at threshold), due to the relatively long lifetime of the upper lasing state $|2\rangle$ (tens of picoseconds) and the relatively fast lifetime of the lower lasing state $|1\rangle$. Because transport across the injector barrier involves the emission of a photon when the 

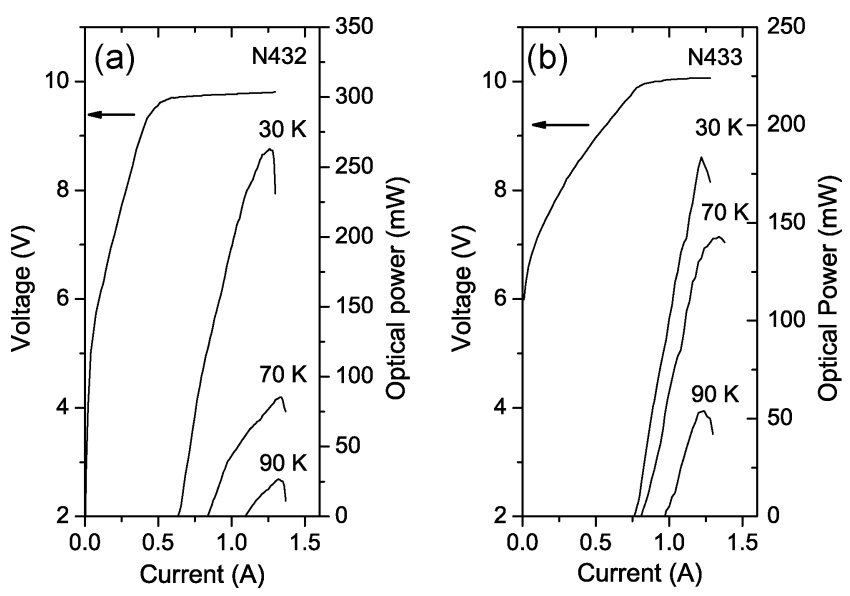

Fig. 2. $\mathrm{CW} L-I-V$ characteristics at various temperatures are displayed for (a) N432 and (b) N433. Note that the observed early voltage clamping near threshold is due to an artifact in the two-point contact measurement of the $I-V$ curve.

system is lasing, the inherent coupling of the electronic transport and lasing action is clearly manifested in this particular structure.

Individual laser bars are processed to have significantly different cavity length and width: laser N432 is $3.53 \mathrm{~mm}$ long and $12.5 \mu \mathrm{m}$ wide, and laser $\mathrm{N} 433$ is cleaved to $2.41 \mathrm{~mm}$ long and $17.1 \mu \mathrm{m}$ wide. The lasers are then soldered on a copper block, wire-bonded, and mounted on a cold finger of a helium-flow vacuum cryostat for the temperature controlled measurements. The measured lasing wavelength slightly above threshold is $5.38 \mu \mathrm{m}$ for $\mathrm{N} 432$ and $5.25 \mu \mathrm{m}$ for $\mathrm{N} 433$, respectively.

These QCLs typically show strong clamping of the voltage at threshold; the measurements of the $\mathrm{CW} L-I-V$ characteristics are illustrated in Fig. 2. We will show the voltage clamping at threshold corresponds to the onset of photon-driven transport in Section IV: once threshold is reached, the voltage and gain remain constant, and the increase in current corresponds to stimulated emission driving the current through the active region of the cascade heterostructure.

\section{B. Time-Resolved Pump-Probe DT}

For the midinfrared (mid-IR) pump-probe DT measurements, mid-IR pulses are generated using an optical parametric amplifier (OPA) and difference frequency generator (DFG), pumped by an $80-\mathrm{fs}, 250-\mathrm{kHz}$ Ti:sapphire regeneratively amplified laser (Coherent RegA). The signal and idler pulses from the OPA are mixed in a $\mathrm{AgGaS}_{2}$ crystal, producing 200 -fs mid-IR pulses tunable from 3 to $7.5 \mu \mathrm{m}$. The pump (800 pJ) and probe $(40 \mathrm{pJ})$ beams are collinearly combined and coupled through the waveguide core of QCL; the coupling efficiency is estimated to be only in the range of $1 \%$, so the pump-induced change in QCL gain is in the small-signal regime. The transmitted probe pulse is collected using f/0.7 optics and measured using a liquid-nitrogen cooled InSb detector. The polarizations of pump and probe beams are orthogonal and linearly polarized at $45^{\circ}$ with respect to the transverse magnetic (TM) lasing mode of a QCL. A polarizer is placed before the detector to block the transmitted and scattered pump beam and suppress coherent artifacts occurring from pump-probe coupling, i.e., interference effects.
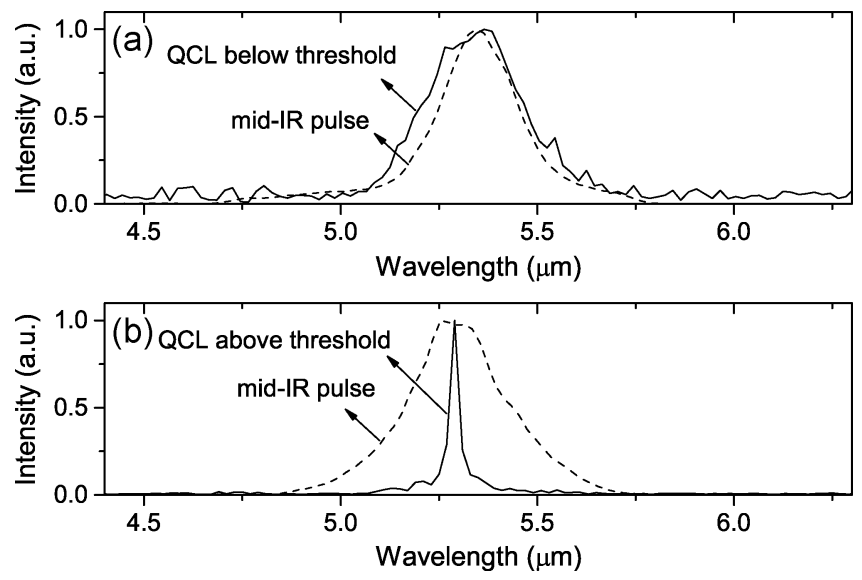

Fig. 3. Luminescence spectra of QCL N433 and those of pump-probe pulses produced by the DFG. Normalized spectra when QCL is biased at (a) $0.67 \mathrm{~A}$ (below threshold) and (b) 0.765 A (slightly above threshold), respectively.

In order to suppress signals coupled through the substrate or cladding, a spatial filter incorporating a $10-\mu \mathrm{m}$ aperture has been carefully positioned to isolate the signal coupled into the waveguide core. The pump pulse is mechanically chopped at $2 \mathrm{kHz}$, and the DT signal is measured using lock-in detection. DT signals as large as $1 \%$ are obtained; the noise floor in these DT experiments is on the order of $0.03 \%$, limited by long-term fluctuations of the laser source and beam pointing. The pump-probe delay is varied using a mechanical stepper stage, and a variable time step is used (smaller steps near zero delay) to reduce data acquisition times. Carrier heating contributions to the DT signal are investigated using transverse electric (TE) pump and TM probe polarizations (see Section VII).

Due to the strong Stark effect in our QCLs, the spectrum of the pump and probe pulses is tuned to match the QCL gain maximum at each bias current: the center wavelength of mid-IR pulse is shifted to shorter wavelength as the bias is increased. Above threshold, the voltage is clamped and the spectrum does not shift with current. Examples of typical QCL and pump-probe pulse spectra are shown in Fig. 3. Below threshold, the electroluminescence spectra are broad, and have a spectral width which closely matches the pump-probe pulse spectral width. Above threshold, the QCL lasing output of course is significantly narrower than the pump-probe spectrum.

\section{RATE-EQUATION MODEL}

In our QCLs, the lasing transition occurs between the upper lasing state $|2\rangle$ and lower lasing state $|1\rangle$, and electrons in the lower lasing state $|1\rangle$ subsequently tunnel into the superlattice miniband state, which is incorporated into the following rate-equation model as a single state $|S L\rangle$. Thus, if we assume no leakage current so that the electrons cascade sequentially through these states in the heterostructure, the laser may be modeled with a three-level model, in which upper lasing state $|2\rangle$ is fed by the superlattice transport, as illustrated in Fig. 4. The rate equations follow directly from the level diagram and appear qualitatively the same as the rate equations for a three-level atomic laser, with one critical difference: following decay out of the lower lasing level, there is a temporal delay before injection into the upper lasing state due to transport 
TABLE I

PARAMETERS USED IN THE RATE-EQUATION MODEL FOR QCL N432 AND N433

\begin{tabular}{c|c|c|c|c|c}
\hline Parameters & Description & Method & $\mathbf{N 4 3 2}$ & $\mathbf{N 4 3 3}$ & Error \\
\hline Lasing wavelength & $\lambda(\mu \mathrm{m})$ & Measured & 5.3 & 5.25 & - \\
\hline Number of cascading stages & $N_{p}$ & Design parameters & 25 & 25 & - \\
\hline Cavity length & $L(\mathrm{~mm})$ & Measured & 3.53 & 2.41 & \pm 0.05 \\
\hline Cavity width & $W(\mathrm{~mm})$ & Measured & 12.5 & 17.1 & \pm 0.25 \\
\hline Waveguide loss & $\alpha\left(\mathrm{cm}^{-1}\right)$ & Estimated & 25 & 25 & \pm 10 \\
\hline Refractive index & $n$ & Calculated & 3.17 & 3.17 & - \\
\hline Reflection coefficient & $r$ & Calculated & 0.27 & 0.27 & - \\
\hline Full width at half maximum & $2 \gamma(\mathrm{meV})$ & Measured & 28 & 28 & \pm 0.5 \\
\hline Matrix element at threshold & $z_{21}(\mathrm{~nm})$ & Measured & 0.33 & 0.38 & - \\
\hline Collection efficiency & $\eta_{c}$ & Assumed & 0.7 & 0.7 & - \\
\hline Spontaneous emission factor & $\beta$ & Measured & $3 \times 10^{-3}$ & $7 \times 10^{-3}$ & - \\
\hline Spontaneous emission lifetime & $\tau_{s p}(\mu \mathrm{s})$ & Calculated & 1 & 1 & - \\
\hline Threshold current & $\mathrm{I}_{\text {th }}(\mathrm{A})$ & Measured & 0.636 & 0.764 & - \\
\hline Confinement factor per period & $\Gamma_{p}(\%)$ & Calculated & 1.88 & 1.84 & - \\
\hline
\end{tabular}

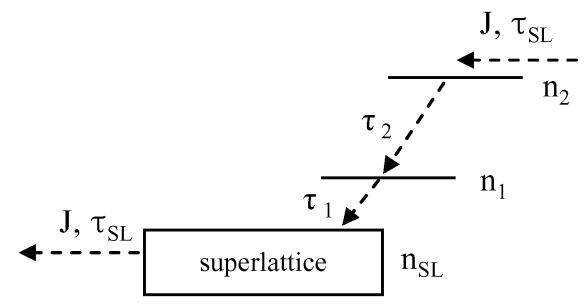

Fig. 4. Three-level model of the active and injector region of a QCL based on diagonal transition; adjacent stages are coupled through the superlattice with a delay $\tau_{S L}$

through the superlattice. This effect has no analogue in an atomic laser or semiconductor interband laser. Note that continuity implies that the current flowing through QCL structure (from the applied bias current) should be same at any point in QCL; $J / q=n_{S L} / \tau_{S L}=n_{2} / \tau_{2}=n_{1} / \tau_{1}$. The rate equations are

$$
\begin{aligned}
\frac{d S}{d t} & =\left[N_{p} \Gamma_{p} v_{g} g_{c}\left(n_{2}-n_{1}\right)-\frac{1}{\tau_{p}}\right] S+N_{p} \beta \frac{n_{2}}{\tau_{s p}} \\
\frac{d n_{2}}{d t} & =\frac{n_{S L}}{\tau_{S L}}-\frac{n_{2}}{\tau_{n r}}-\Gamma_{p} v_{g} g_{c}\left(n_{2}-n_{1}\right) S \\
\frac{d n_{1}}{d t} & =\frac{n_{2}}{\tau_{n r}}-\frac{n_{1}}{\tau_{1}}+\Gamma_{p} v_{g} g_{c}\left(n_{2}-n_{1}\right) S \\
\frac{d n_{S L}}{d t} & =\frac{n_{1}}{\tau_{1}}-\frac{n_{S L}}{\tau_{S L}}
\end{aligned}
$$

where $n_{2}, n_{1}, n_{S L}$ are the electron density in $|2\rangle,|1\rangle,|S L\rangle$, and $S$ is the photon density. $N_{p}$ is the number of stages in the QCL, $\Gamma_{p}$ is the confinement factor for one period, $v_{g}$ is the group velocity, $g_{c}$ is the gain cross section, $\tau_{p}$ is the photon lifetime, $\beta$ is the spontaneous emission factor (fraction of spontaneous emission emitted into the lasing mode), $\tau_{s p}$ is the spontaneous emission lifetime, $\tau_{S L}$ is the superlattice transport time, $\tau_{n r}$ is the nonradiative lifetime of $|2\rangle$, and $\tau_{1}$ is the tunneling time from |1) to the superlattice.

The complete set of parameters used to model the two QCL lasers investigated in this study are shown in Table I. This model is used to fit the DT data reported in Section IV, and the time constants are extracted from these fits. We emphasize that three time constants are required for good fits to the data, which is a direct consequence of the three-level nature of the diagonal QCL operation, as dicussed in Section IV.

An additional check on the validity of the model is obtained through calculation of the threshold current $J_{\text {th }}$ : we replace $n_{S L} / \tau_{S L}$ with $J / q$ in order to establish a steady-state current in the device. The threshold current neglecting spontaneous emission and lower state emptying (several orders faster than the upper state decaying) is derived by setting the time-derivatives in the rate-equations to zero, and the other QCL parameters yield [23]

$$
\begin{aligned}
J_{\mathrm{th}} & \simeq q \cdot \frac{\alpha_{\text {mirror }}+\alpha_{\text {waveguide }}}{N_{p} \Gamma_{p} g_{c} \tau_{n r}} \\
S & =2 \frac{P \tau_{p}}{h \nu \eta_{c} L W} \\
\tau_{2, \text { threshold }} & \simeq \tau_{s t} \simeq \frac{N_{\text {inversion }}}{v_{g} g_{c} N_{\text {doping }} N_{p}} \cdot \frac{1}{S / L W} \\
g_{c} & =\frac{1}{L_{p}} \cdot \frac{4 \pi q^{2} z_{21}^{2}}{\epsilon_{o} n \lambda 2 \gamma} .
\end{aligned}
$$

In order to obtain a self-consistent set of parameters for modeling the QCL operation, we use the following procedure. From the measured $L-I$ curve, we determine the threshold current density $J_{\mathrm{th}}$. From the DT data at threshold, we determine the upper state lifetime. Since the current is related to the lifetime be $J=q N / \tau$, the density $N$ is determined (with a resulting value close to the design value of the doping density). The $L-I$ curve also allows us to deduce the intracavity photon density (the uncertainty being determined mainly by our estimate of the detector collection efficiency, assumed to be $\sim 70 \%$ ). Combining the upper state density and photon density, we can get the matrix element $z_{21}$ at threshold; the resulting value of $0.33 \mathrm{~nm}$ is slightly below the calculated design value of $0.38 \mathrm{~nm}$, but they are all within the errors arising from the monolayer fluctuations. The self-consistency of the parameters in the rate-equation model is checked by recalculating the threshold current density: the calculated $J_{\text {th }}$ are $1.45 \mathrm{kA} / \mathrm{cm}^{2}$ for $\mathrm{N} 432$ and $1.88 \mathrm{kA} / \mathrm{cm}^{2}$ for $\mathrm{N} 433$, which is in excellent agreement with the measured values of $1.44 \mathrm{kA} / \mathrm{cm}^{2}$ for $\mathrm{N} 432$ and $1.85 \mathrm{kA} / \mathrm{cm}^{2}$ for $\mathrm{N} 433$. Note that the error for the threshold current calculation arises from QCL design parameters and measurement uncertainties (e.g., waveguide loss, cavity length and width, and transition FWHM), which is around 10\%-15\%. 


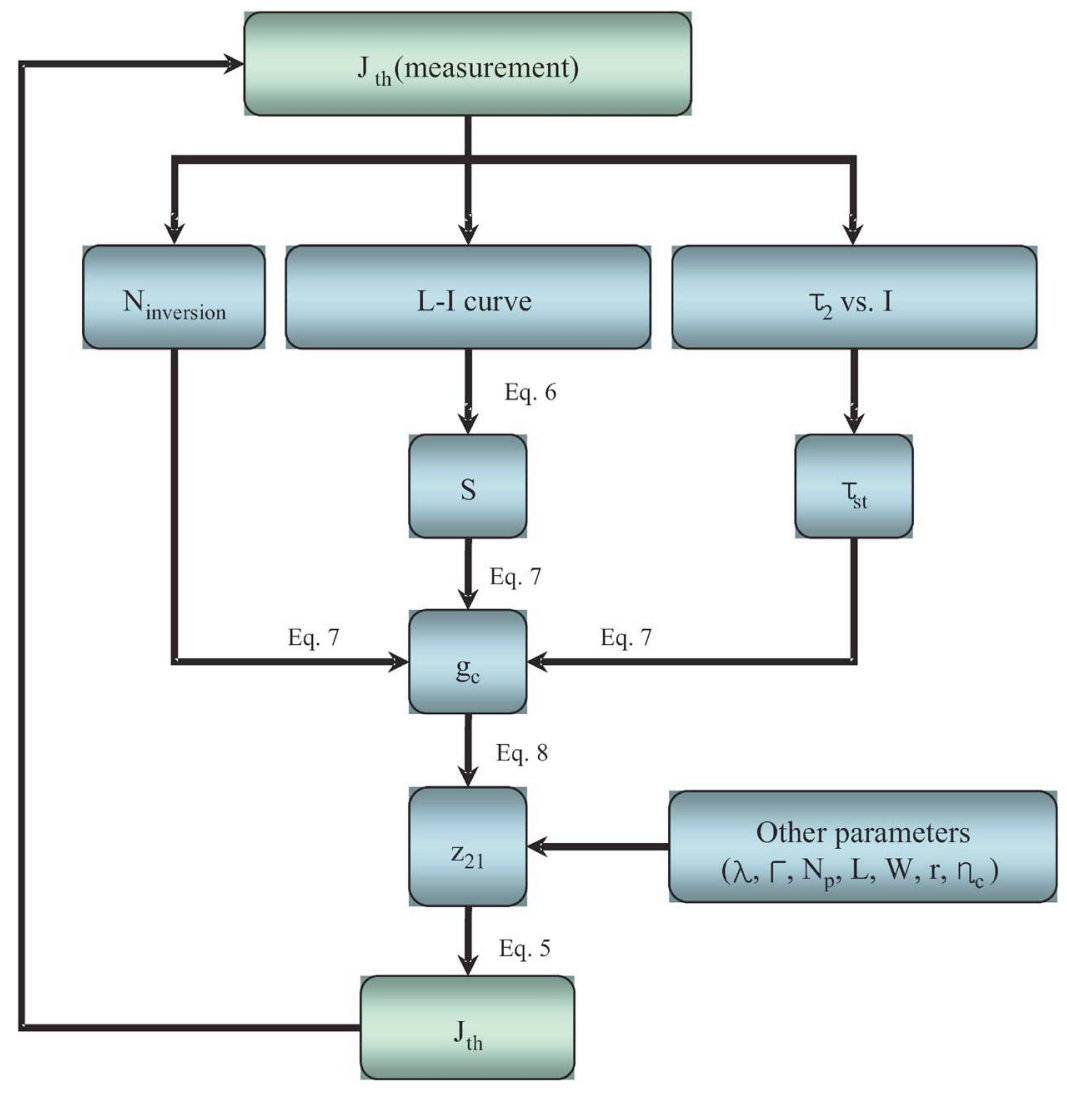

Fig. 5. Flow diagram to calculate relevant QCL parameters.

A schematic of flow diagram for the consistent check of $J_{\text {th }}$ and $z_{21}$ is displayed in Fig. 5 .

\section{GAIN ReCOVERY DyNAMICS IN QCLS}

\section{A. Photon-Driven Transport}

As discussed in Section II, the lasers used in this study are based on a "diagonal transition" in real space. Although a population inversion is present at all bias fields in these experiments, the cavity gain is controlled by voltage tuning of the oscillator strength through the linear Stark effect. In these structures, the lowest state of the injector region serves as both the electron reservoir and the upper lasing state. Laser action takes place between the upper lasing state $|2\rangle$ and the lower lasing state $|1\rangle$ in the active region, which is emptied via tunneling into the superlattice. Before an electron contributes to stimulated emission in the next active region, it must drift across the superlattice injector region.

As can be seen in the rate equation in Section III, there are four processes that enter the dynamics: 1) phonon-assisted relaxation out of upper lasing state $|2\rangle$;2) stimulated emission out of upper lasing state $|2\rangle ; 3)$ depletion of lower lasing state; and 4) transport across the superlattice. We will consider each of these processes in the following.

First, we consider how the current is driven by the laser photon density in such a structure. In a cascade heterostructure, the current through a given level is given by $J=q N / \tau$, where $q$ is the electron charge, $N$ is the areal density, and $\tau$ is the state lifetime. Below threshold, the $|2\rangle$ lifetime is determined by (off-resonant) phonon-assisted tunneling (in the 20-50-ps range in our QCLs). Above threshold, the relevant lifetime should become the stimulated emission lifetime $\tau_{\mathrm{st}}$.

This simple picture has inspired the study of electronic transport to be studied via the dynamic gain depletion and recovery with the QCL operating in continuous wave at different values of current close to the threshold. The center wavelengths of the pump and probe are tuned to be resonant with the gain transition at each bias. In Figs. 6 and 7, selected bias-dependent DT results at $30 \mathrm{~K}$ are displayed. Qualitatively similar recovery dynamics are observed in both lasers; a three-level model for the cascade structure, coupled to a rate-equation for the intracavity photon density, provides good fits to the data, with only slight differences in the time constants obtained. For positive pump-probe delay, negative DT signals due to the depletion of gain by the pump are observed at all bias currents. The recovery of the DT can be understood as the gain recovery due to electron transport in the cascade heterostructure following the pump-induced gain depletion.

The gain recovery is fit by solving a three-level rate-equation model presented in Section III including coupling to a singlemode-cavity photon flux, and the results are shown as the dashed lines in Figs. 6 and 7. We find that fits using fewer than three temporal components are unable to systematically fit the DT curves.

Note that, below threshold, the DT is obviously less than the DT at and above threshold, since the gain is low. At threshold and above, the gain should be constant, since the gain should in principle clamp at threshold, and this would lead to a constant 
(a)
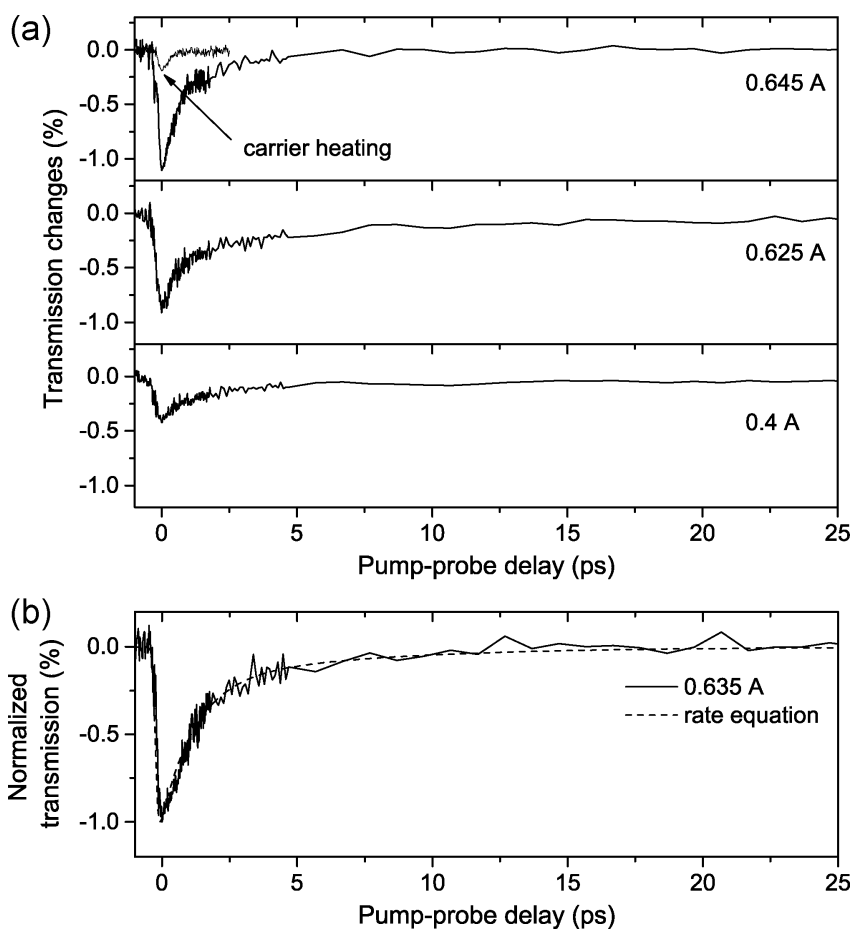

Fig. 6. DT measurements of the gain recovery at $30 \mathrm{~K}$. (a) Representative DT data for QCL from N432 at bias currents 0.4, 0.625, and $0.645 \mathrm{~A}$, respectively. Hot carrier effect is experimentally shown to be negligible (see Section VII). (b) A fit to the three-level rate-equation model (dashed line) of the normalized DT signal at $0.635 \mathrm{~A}$ (solid line) is shown.

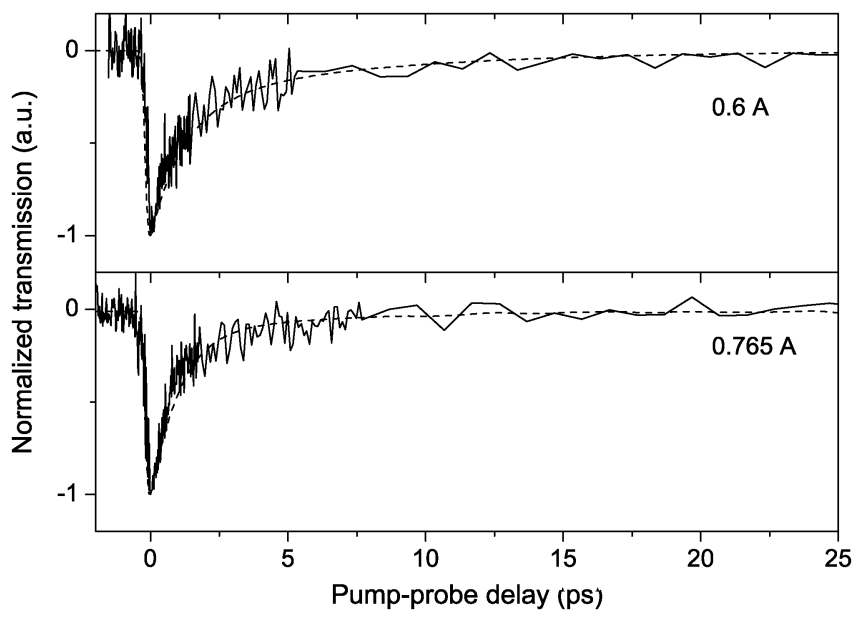

Fig. 7. Bias-dependent DT signals at $30 \mathrm{~K}$ for N433. Upper and lower panel are at 0.6 and $0.765 \mathrm{~A}$, respectively. Dashed lines show the results from the rate-equation fit.

DT amplitude versus bias above threshold. We have observed that there are very slight changes (less than $0.1 \%$ ) of DT at timezero delay as the bias is increased above threshold. However, in this QCL as in nearly all QCLs, the voltage does not perfectly clamp above threshold, and this yields a slight increase of the DT above threshold.

The key result of this section is shown in Fig. 8(a) and (b), which display the upper state $|2\rangle$ lifetime $\tau_{2}$, obtained from the rate-equation fits of the DT data, as a function of bias current. We consider three processes contributing to the decay of the upper state, namely phonon-assisted intersubband relaxation
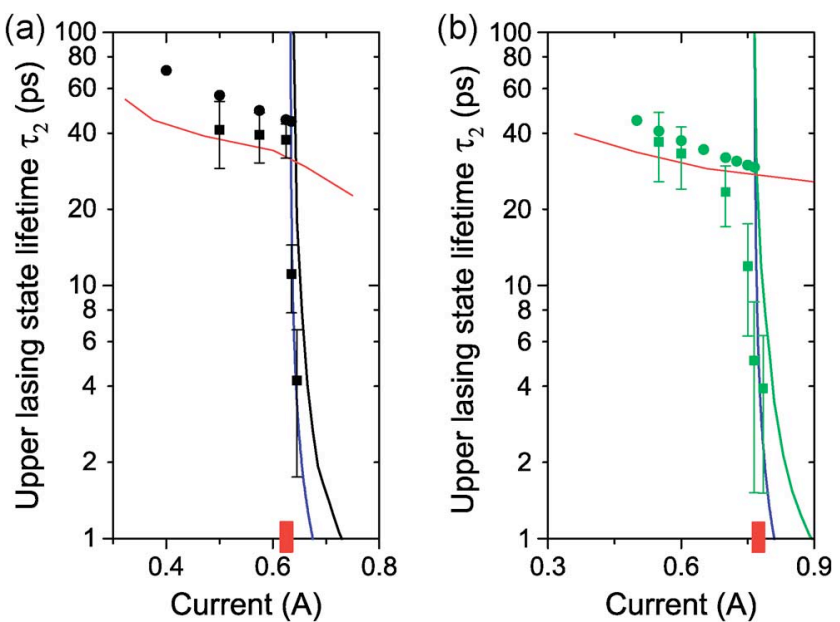

Fig. 8. Bias-dependent upper state $|2\rangle$ lifetime $\tau_{2}$ is shown for (a) N432 and (b) N433 (threshold is indicated by the red tick mark). The filled squares are 12) lifetime obtained from rate-equation fits to the gain recovery DT data. The red solid lines are $|2\rangle$ lifetime due to optical-phonon scattering calculated using the bias-dependent wavefunctions; the errors obtained by the monolayer fluctuations of the barrier width are around $10 \mathrm{ps}$, which is within our measurement. The filled circles are an estimate of $|2\rangle$ lifetime using $J=q N / \tau_{2}$ and assuming the $|2\rangle$ population $N$ is simply the doping density (which has a likely error in the 20\%-40\% range). The dramatic reduction in lifetime due to the onset of stimulated emission just below threshold corresponds to the current through the QCL being limited by the photon-driven transport across the QCL active region. Vertical blue and black solid lines in (a) and blue and green solid lines (b) are stimulated emission lifetimes (see the main text).

[24] (with time constant $\tau_{\mathrm{ph}}$ ), radiative decay by spontaneous emission $\left(\tau_{\mathrm{sp}}\right)$, and stimulated emission $\left(\tau_{\mathrm{st}}\right)$. The total upper state $|2\rangle$ lifetime can be written as $\tau_{2}^{-1}=\tau_{\mathrm{ph}}^{-1}+\tau_{\mathrm{sp}}^{-1}+\tau_{\mathrm{st}}^{-1}$. In this expression, the radiative decay is negligible because $\tau_{\mathrm{sp}}$ is several microseconds. When the bias is well below threshold, the upper state $|2\rangle$ lifetime is essentially $\tau_{p h}$ ranging around $20-50 \mathrm{ps}$ (decreasing slightly with bias due to the change in wavefunction overlap). As the QCL bias voltage approaches threshold, stimulated transitions start to occur, reducing the upper state $|2\rangle$ lifetime and increasing the current.

The stimulated emission lifetime $\tau_{\text {st }}$ is obtained in two ways. First, $\tau_{\mathrm{st}}$ is calculated directly by obtaining the cavity photon density $S$ from the measured output power (solid line in Fig. 9) and using $\tau_{s t}=N / v_{g} N_{p} g_{c} S$, where $N$ is the population inversion (assumed to be the doping density), $v_{g}$ is the group velocity, $N_{p}$ is the number of cascading stages, and $g_{c}$ is the gain cross section; the result is shown as the black and green solid vertical line in (a) and (b), respectively. Second, the rate equation is used to fit the $L-I$ curves (Fig. 9), and the resulting $S$ are used to calculate $\tau_{\text {st }}$. The result is the blue solid vertical line in Fig. 8(a) and (b). In those figures, a dramatic reduction of the upper state lifetime as the QCL approaches threshold is very clear for both devices. Above threshold, $\tau_{\text {st }}$ becomes of an order of a few picoseconds and does not appear in the DT gain recovery dynamics (i.e., above threshold the DT dynamics are determined entirely by $|1\rangle$ emptying and the superlattice transport component).

In conventional atomic or molecular lasers, or of interband semiconductor lasers, the gain recovery time is the upper state spontaneous emission lifetime $\tau_{\mathrm{sp}}$ when the laser is below threshold, and it is $\tau_{\mathrm{sp}} / r$ in a short-cavity lifetime limit when 


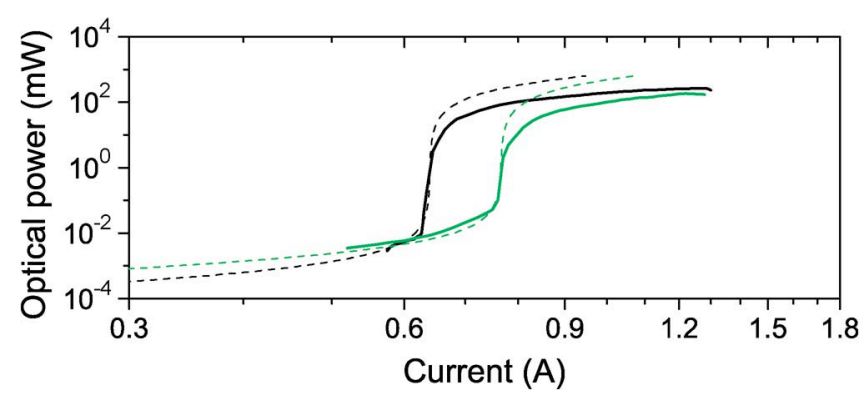

Fig. 9. Rate-equation fits (dashed line) to the measured $L-I$ curves (solid line) at $30 \mathrm{~K}$ are shown. Black and green color are for N432 and N433 QCL, respectively. The only free parameter required to fit the $L-I$ curves with the rate-equation model is $\beta$.

the laser is biased above threshold, where $r$ is the ratio of the pump rate to the threshold pump rate [23]. (The short-cavity limit is used to eliminate the relaxation oscillations which would otherwise mask the gain recovery.) However, the gain recovery dynamics in a QCL, as displayed in Fig. 8, shows an order-of-magnitude speed-up in the contribution of the upper state lifetime to the gain recovery as the bias approaches threshold.

The most important factor leading to the dramatic reduction in upper state lifetime is the interplay of the non-radiative nature of the upper state decay in a QCL far below threshold, and the onset of stimulated emission near threshold. Far below threshold, the phonon-assisted lifetime is weakly bias-dependent (a consequence of the Stark tuning of the wavefunctions). Calculations using the rate-equation model show that, just below threshold, the photon density in the cavity becomes of the order of 100 , which is sufficient to drive the stimulated emission lifetime down to a value comparable to the nonradiative lifetime. It is also an important feature of QCLs that the $\beta$ factor, i.e., the fraction of spontaneous emission emitted into the lasing mode, is rather high, approximately $10^{-3}$, as obtained from the $L-I$ curves. As the current increases above threshold, the upper state lifetime decreases due to the stimulated emission, but it no longer appears in the gain recovery since the gain recovery is limited in that case only by the lower state tunneling and superlattice transport delay.

For the self-consistency of the model used to understand the gain recovery dynamics, the rate-equation model is verified by the following procedure. First, we calculate $\tau_{s t}$ via the threelevel rate-equation model [blue solid line in Fig. 8(a) and (b)], finding good agreement with the DT data. Second, we calculate $\tau_{\text {st }}$ via an estimate of the intracavity photon density obtained from the measured steady-state $L-I$ characteristic; this appears as the black and green solid line in Fig. 8(a) and (b), respectively. Finally, the rate equations used to fit the dynamics are solved in steady-state to obtain the threshold current and steady-state $L-I$ curves as explained in Section III; these are seen in Fig. 9 to agree quite well with the experiment.

\section{B. Gain Dynamics in Four-Level Conventional Lasers}

We have noted above that the QCL rate equations differ from those of atomic systems or interband semiconductor lasers in an important way: following stimulated emission, there is a delay

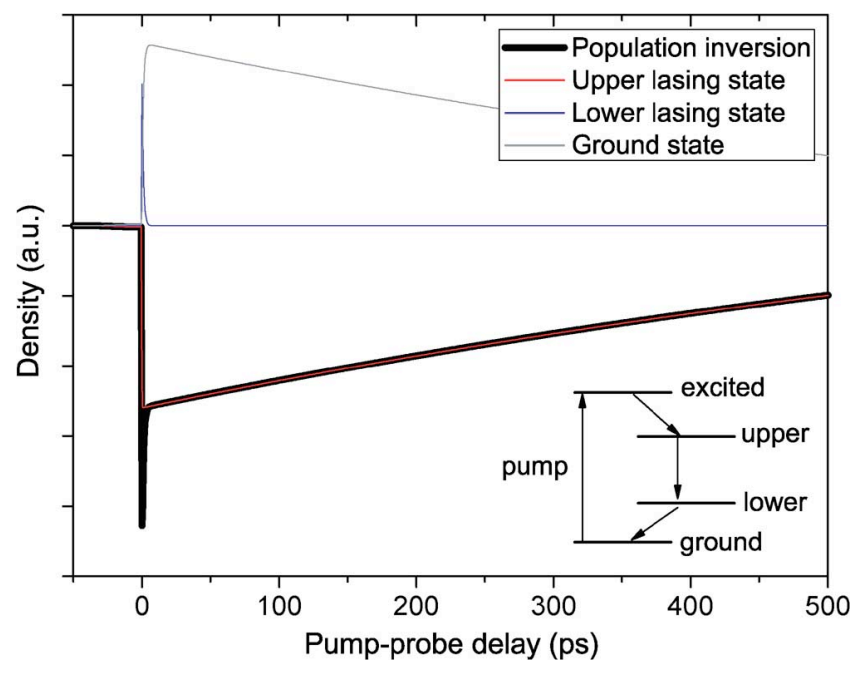

Fig. 10. Rate-equation analysis for pump-probe measurement on a four-level atomic solid-state laser. Note that the four-level laser system is effectively a closed system in that there is no transport delay for the carrier pumping.

in the injection of the electron into the upper lasing state due to transport through the superlattice. It is this feature (along with the large $\beta$ factor of QCL) which enables the photon-driven transport to appear in the gain recovery dynamics. In order to illustrate this point, we present here the solution for the gain recovery dynamics in a model atomic rate-equation system. We assume a standard four-level model appropriate for an atomic (or interband laser diode) system [23]. In most such lasers, the gain recovery dynamics will be complicated by relaxation oscillations due to the comparatively long upper state lifetime. In order to suppress these relaxation oscillations in the model and thus facilitate a more direct comparison, we take the cavity lifetime in the four-level model to be artificially short, namely $100 \mathrm{fs}$. We assume an upper state lifetime of $1 \mathrm{~ns}$ and a lower state lifetime of $1 \mathrm{ps}$. The result of a numerical integration of the rate equations is shown in Fig. 10, with the laser initially oscillating in the steady state, and a 220 -fs pulse resonant with the gain transition is injected at time-zero. A two-component gain recovery is observed with the fast component of the gain recovery being the lower state lifetime and the long component determined by the upper state lifetime.

\section{Relation of the Three-Level Model to Previous Transport Models}

Previous investigations of transport in QCLs have been based on steady-state analysis of the $L-I$ and $V-I$ curves of the devices [25]. In that analysis, a two-level rate-equation model was applied, with the two relevant time constants being the upper and lower lasing state lifetimes. By comparing the differential conductance from the $I-V$ curves above and below threshold, the lower state $|1\rangle$ lifetime of $2.5 \mathrm{ps}$ was deduced. We note here that the two-level model applied in that work can essentially be considered a simplification of the three-level model applied here.

In our model, there are three relevant processes: upper state $|2\rangle$, lower state $|1\rangle$ lifetimes, and superlattice $|S L\rangle$ delay time. The two-level model effectively neglects the superlattice transport. We have carried out analysis similar to that of [25], but with 
the three-level model. In a similar fashion to [25], the three-level rate-equation model can be written as

$$
\begin{aligned}
\frac{d n_{2}}{d t} & =\frac{n_{S L}}{\tau_{S L}}-\frac{n_{2}}{\tau_{2}}-g_{c} S\left(n_{2}-n_{1}\right) \\
\frac{d n_{1}}{d t} & =\frac{n_{2}}{\tau_{2}}-\frac{n_{1}}{\tau_{1}}+g_{c} S\left(n_{2}-n_{1}\right) \\
\frac{d n_{S L}}{d t} & =\frac{n_{1}}{\tau_{1}}-\frac{n_{S L}}{\tau_{S L}}
\end{aligned}
$$

where the product of gain cross section and lifetimes are assumed to be constant

$$
g_{c} \tau_{2}=\tilde{g} \tau_{1}=\check{g} \tau_{S L}=A
$$

and an ideal gain cross section $g_{0}$ for zero lower state lifetime is defined as

$$
g_{0}\left(n_{2}+n_{1}+n_{S L}\right)=\text { total loss. }
$$

After solving the rate equation at steady state, one can obtain the photon-flux density $S$ as

$$
S=\frac{\check{g} \tilde{g} g_{c}-g_{0} \check{g} \tilde{g}-\check{g} g_{c}^{2}-\check{g} g_{0} g_{c}-\tilde{g} g_{0} g_{c}}{A g_{0} g_{c}\left(\tilde{g}-g_{c}\right)} .
$$

The differential conductance below threshold $G_{b}$ and above threshold $G_{a}$ are given by

$$
\begin{aligned}
G_{b} & =\frac{\tilde{g}^{2} \breve{g}^{2}}{\left(\tilde{g} \check{g}+\tilde{g} g_{c}+g_{c} \check{g}\right)^{2}} \\
G_{a} & \simeq \frac{\check{g}^{3} g_{0}}{2 g_{0}^{2} \check{g}^{2}}=\frac{\check{g}}{2 g_{0}}
\end{aligned}
$$

and the ratio of the differential conductance $G_{b} / G_{a}$ is

$$
\frac{G_{b}}{G_{a}}=\frac{\frac{\check{g}}{2 g_{0}}}{\frac{\tilde{g}^{2} \check{g}^{2}}{\left(\tilde{g} \check{g}+\tilde{g} g_{c}+g_{c} \check{g}\right)^{2}}} \simeq \frac{1}{2} \frac{\check{g}}{g_{0}} .
$$

The main conclusion of the above analysis is that, if one lumps the $|1\rangle$ lifetime and $|S L\rangle$ transport processes together in order to transform the three-level model into a two-level one, then the ratio of the differential conductance reveals the slower of those two time constants (as long as one of the time constants is significantly shorter than the other). In our system, the DT experiments show that the lower state $|1\rangle$ lifetime is approximately $0.7 \mathrm{ps}$, and the superlattice transport time is about $2 \mathrm{ps}$. The 2-ps time constant obtained from DT is therefore consistent with steady-state transport characterization, but the DT experiments enable us to refine the QCL transport model and separate the lower state lifetime and superlattice transport processes.

\section{Dynamics of the LOWER Lasing State}

In addition to the upper state $|2\rangle$ lifetime, two other components are observed in the gain recovery dynamics. In this section, we discuss the observed fastest component $\tau_{1}$, which is attributed to the lower state emptying.

The time constant of $\tau_{1}$ is approximately $0.7 \mathrm{ps}$ from a rateequation fit and corresponds to the decay of lower lasing state
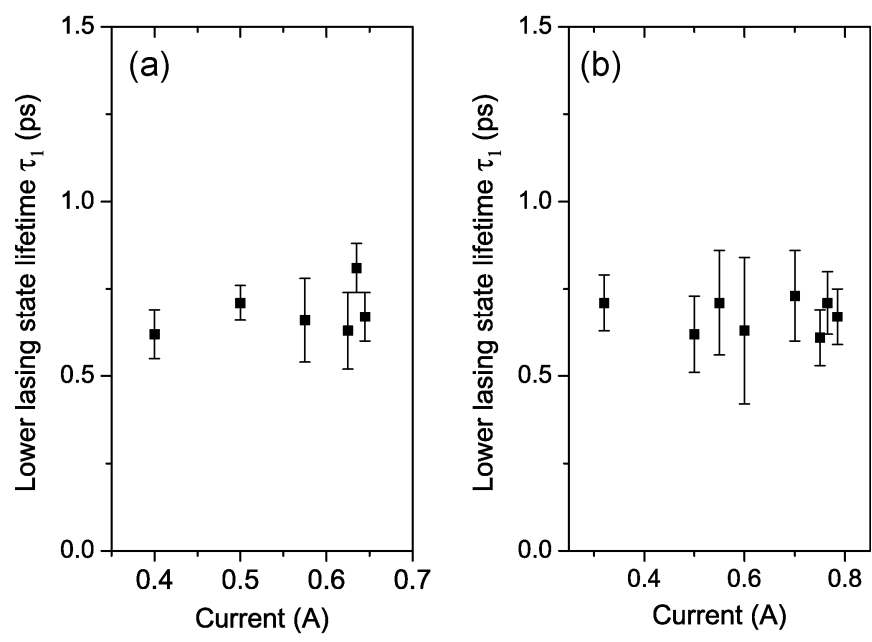

Fig. 11. Extracted $|1\rangle$ lifetimes from the rate-equation fit for (a) N432 and (b) $\mathrm{N} 433$.

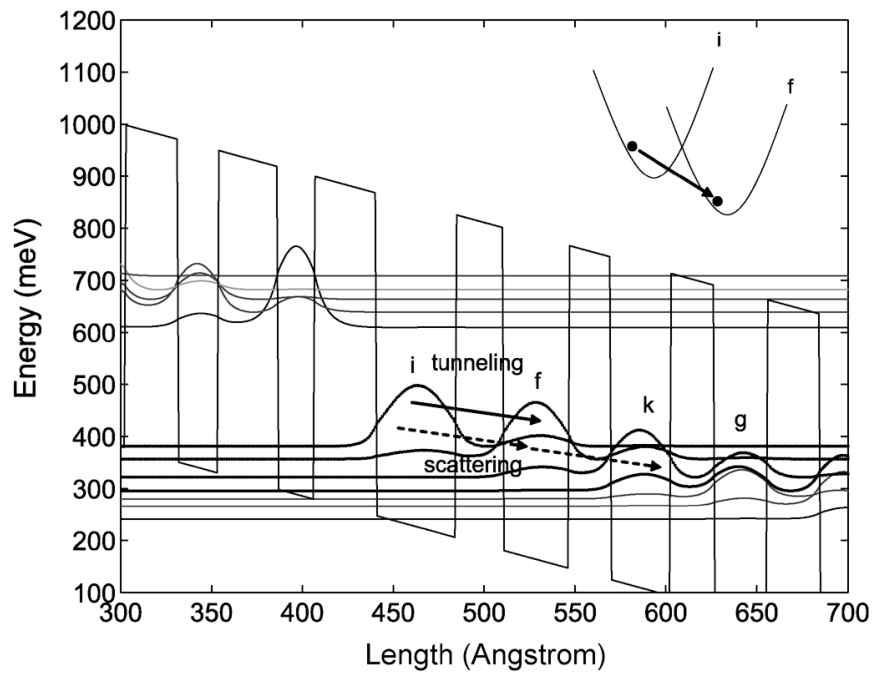

Fig. 12. Schematic bandstructure diagram of N432 to explain the lower state emptying due to tunneling and scattering.

$|1\rangle$. This relaxation across the barrier into the superlattice is controlled by electron tunnelling in the presence of strong scattering including the electron-polar-optical-phonon interaction [26], [27] and interface roughness scattering, leading to an expected relaxation time around $0.7 \mathrm{ps} ; 1 / \tau_{1}=1 / \tau_{\text {tunneling }}+$ $1 / \tau_{\text {scattering. }}$. This is much faster than any other process that may possibly contribute to the gain recovery. Contributions from carrier heating and waveguide anisotropy are experimentally shown to be small (see Section VII). The DT rate-equation fits to the bias-dependent $\tau_{1}$ lifetime are summarized in Fig. 11. This relaxation process is largely bias-independent since the final density of states through the barrier downstream is always high and nearly independent of bias.

Fig. 12 shows a schematic diagram for the lower state emptying due to the tunneling and scattering. We first estimate the tunneling rate by the following way. Two-level density matrix equations are used to derive the tunneling rate based on a tightbinding approximation between initial state $|i\rangle$ and final state 

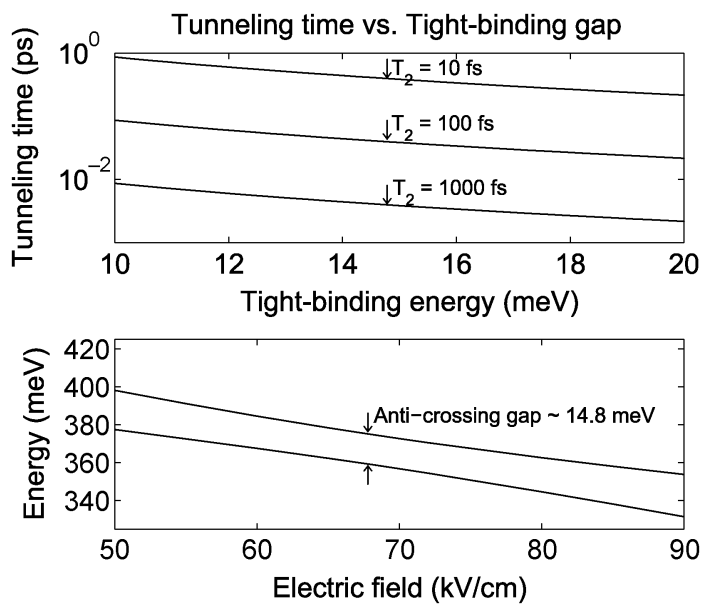

Fig. 13. Tight-binding model for the coupled QW systems of Fig. 12 to estimate the tunneling rate out of the lower lasing state.

$|f\rangle$. The equations of motion for the coupled tunneling systems is described by [28]

$$
\begin{aligned}
\frac{d \rho_{i i}}{d t} & =\frac{i \Omega_{R}}{2}\left(\rho_{f i}-\rho_{f i}^{*}\right)-\frac{\rho_{i i}}{\tau_{i}}-\frac{\rho_{i i, 0}}{\tau_{i}} \\
\frac{d \rho_{f f}}{d t} & =-\frac{i \Omega_{R}}{2}\left(\rho_{f i}-\rho_{f i}^{*}\right)-\frac{\rho_{f f}}{\tau_{f}}-\frac{\rho_{f f, 0}}{\tau_{f}} \\
\frac{d \rho_{f i}^{*}}{d t} & =-\frac{i}{\hbar}\left[\Delta E \rho_{f i}^{*}+\frac{\Omega_{R} \hbar}{2}\left(\rho_{i i}-\rho_{f f}\right)\right]-\frac{\rho_{f i}^{*}}{\tau_{\|}} \\
\frac{d \rho_{f i}}{d t} & =\frac{i}{\hbar}\left[\Delta E \rho_{f i}+\frac{\Omega_{R} \hbar}{2}\left(\rho_{i i}-\rho_{f f}\right)\right]-\frac{\rho_{f i}}{\tau_{\|}}
\end{aligned}
$$

where $\rho_{i i}$ and $\rho_{f f}$ are the diagonal elements of the state population and $\rho_{f i}$ is the off-diagonal element of the mixed state. $\Delta E$ is the energy detuning between state $|i\rangle$ and $|f\rangle, 1 / \tau_{\|}$is the dephasing rate in the tunneling process, and $\rho_{i i, 0}-\rho_{f f, 0} \simeq$ $\rho_{i i, 0}=1$. The energy splitting $\hbar \Omega_{R}$ is the anti-crossing energy gap, which can be calculated when the two coupled states are in resonance.

Following a standard procedure as given in [29]-[31], the tunnel-assisted lower state emptying rate is expressed as

$$
\frac{1}{\tau_{\text {tunneling }}}=\frac{\Omega_{R}^{2} \frac{1}{2}\left(\frac{1}{\tau_{\|}}\right)}{\left(\frac{1}{\tau_{\|}}\right)^{2}+\left(\frac{\Delta E}{\hbar}\right)^{2}} .
$$

As shown in Fig. 13, given the dephasing time $\tau_{\|}$around a few tens of femtoseconds, the computed tunneling rate based on the (22) is approximately two to three times larger than the measurement results.

The second contribution is the scattering-assisted emptying. Among the various scattering mechanisms, we consider only the electron-polar-optical-phonon scattering process; in the intersubband transition, the dominant scattering process is usually limited by the longitudinal polar-optical-phonon emission rate. Based on the Fermi golden rule, the average phonon scattering rate from initial state $|i\rangle$ to final state $|f\rangle$ reads

$$
\left(\frac{1}{\tau_{i f}}\right)_{\mathrm{avg}}=\frac{\int W_{i f}^{\mathrm{ph}, \mathrm{em}} f_{i}(E) d E}{\int f_{i}(E) d E}
$$

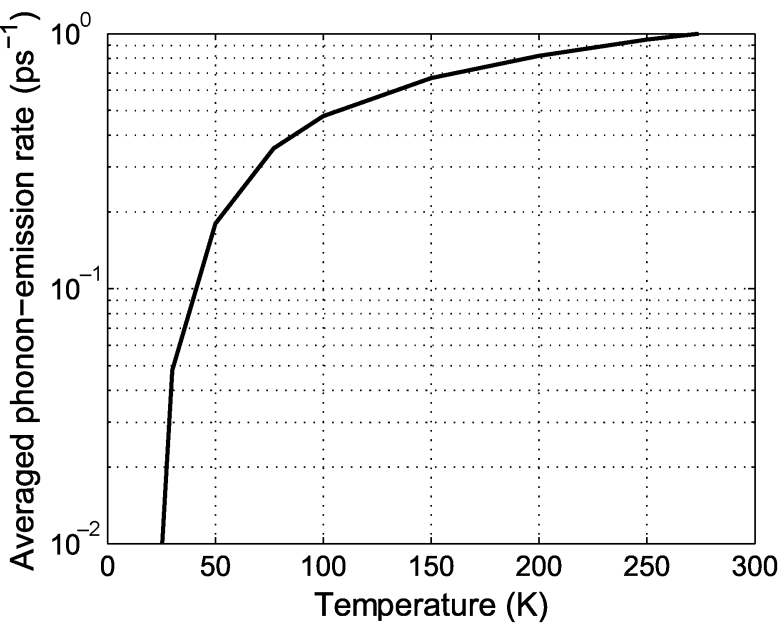

Fig. 14. Temperature dependence of the average electron-phonon scattering rate, assuming an electron temperature is same as the lattice temperature. Again, note that the electron temperature is expected to be than the heat sink temperature of $30 \mathrm{~K}$.

where $W_{i f}^{\text {ph,em }}$ is the the longitudinal polar-optical-phonon emission rate based on a single particle picture and $f_{i}(E)$ is the Fermi-Dirac distribution function [32].

The average phonon emission rate assuming the electron temperature is the same as the lattice temperature is displayed in Fig. 14; the experimentally relevant scattering rate is the average phonon scattering. Considering the lattice temperature is always higher than the heat sink temperature [33], the calculated tunneling rate is approximately two to three times smaller than the measurement results.

From the calculations on the tunneling rate and phonon emission rate, we attribute the total lower state dynamics to be governed by a sum of the two processes

$$
1 / \tau_{1}=1 / \tau_{\text {tunneling }}+1 / \tau_{\text {scattering }} .
$$

The calculated total lower state dynamics is in the range of $1 \mathrm{ps,}$ which qualitatively agrees with the DT measurement result.

\section{Dynamics of SuPERLATtice RELAXation}

The third time constant contributing to the gain recovery dynamics is associated with the superlattice transport. In the steady state, the relevant time constant in the rate equations, $\tau_{S L}$ is the superlattice drift time. In a dynamics experiment, however, the time constant for the charge distribution to return to equilibrium following an impulsive perturbation (in our case, the stimulated emission across the active region due to the pump pulse) is not the drift time across the entire superlattice. Instead, it is the dielectric relaxation time.

A rigorous understanding of the dynamical superlattice relaxation would require a full quantum-mechanical model coupled to Poisson's equation, with hot carrier effects included at the same level [33]. However, if the field across the superlattice injector is sufficiently low that the transport can be described in a low-field transport regime (mobility regime), then one can apply an approximate model to understand the essential features.

Our approach is to use a simple dielectric relaxation model which is based on the calculation of the changes of nonuniform 
electron density following the pump-pulse-induced impulsive excitation in a superlattice.

In a bulk dielectric relaxation model, the temporal solution of a coupled 1-D current continuity and Poisson equation gives the exponential decay of the local carrier fluctuation [34]

$$
n-n_{0}=\left(n-n_{0}\right)_{t=0} \exp \left(-t / \tau_{d}\right)
$$

where $n-n_{0}$ is the local carrier density fluctuation from equilibrium. The dielectric relaxation time $\tau_{d}$ is given by

$$
\tau_{d}=\epsilon / \sigma=\epsilon / q \mu N
$$

where $\sigma$ is the conductivity, $\mu$ is the mobility for the transport, and $N$ is the background carrier density. If the carrier transport can be described in a linear transport regime, then (26) shows the dielectric relaxation is a function of the material's conductivity, which is controlled by the mobility $\mu$.

The standard bulk dielectric relaxation model, however, is inadequate due to the nonuniform carrier density in the superlattice. We have modified the model in the following way. First, a simple serial resistor model is used to calculate the voltage drops (electric fields) across the active and injector region. Since the DT dynamics is a function of the applied bias current, the biasdependent electric field distributions are qualitatively estimated. Then, a Monte Carlo simulation is used to estimate the field-dependent background electron density. Given the electric field distributions and background electron density, the Esaki-Tsu model [35] is used to estimate the superlattice mobility $\mu$; the mobility is also checked using the QCL design parameters.

\section{A. Inhomogeneous Electric Field}

The electric-field changes after the perturbation are calculated based on the following simple resistor-network model. Assuming no leakage current, the QCL can be modeled as a serial circuit comprised of two coupled resistors: active region and superlattice injector region. For simplicity, we neglect the space-charge effect in the superlattice injector; the function of the doped region may be modeled as a capacitor, but it is well known that the space-charge effect in QCLs is negligible [18]. Since each resistor has its own resistance proportional to the state lifetime, the voltage drops across the active and injector region, i.e., the electric field distributions, can be obtained at each bias current.

From current continuity, the current flowing in the QCL should be same in each region $\left(I_{\mathrm{QCL}}=I_{\text {active }}=I_{\text {injector }}\right)$, and therefore the voltage drop across each resistor may be expressed as

$$
\begin{array}{r}
I_{\mathrm{QCL}}=\frac{V_{\text {active }}}{R_{\text {active }}}=\frac{V_{\text {injector }}}{R_{\text {injector }}} \\
\rightarrow \frac{V_{\text {active }}}{V_{\text {injector }}}=\frac{R_{\text {active }}}{R_{\text {injector }}}=\frac{\tau_{\text {active }}}{\tau_{\text {injector }}} .
\end{array}
$$

Assuming that $\tau_{\text {active }}=\tau_{2}$ and $\tau_{\text {superlattice }}=\tau_{S L}$, where $\tau_{2}$ and $\tau_{S L}$ are the upper state lifetime and superlattice relaxation from the rate-equation fits to the experimentally measured DT (a)

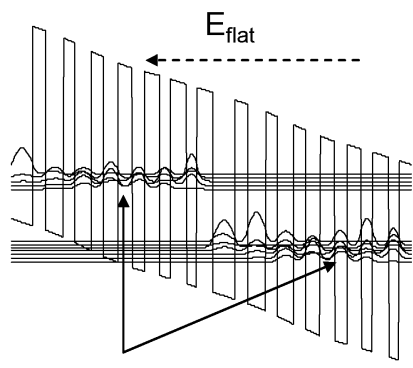

flat miniband (b)

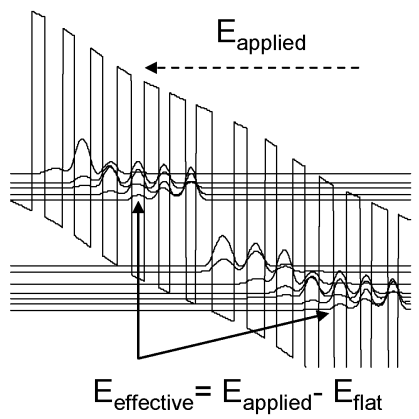

Fig. 15. Schematic conduction band profile for N432 QCL to illustrate an effective electric field in superlattice. (a) Flat band condition at $52 \mathrm{kV} / \mathrm{cm}$. (b) Lasing condition at $95 \mathrm{kV} / \mathrm{cm}$.

data, the voltage drops (and the corresponding electric fields) across the active region and the superlattice region read

$$
\begin{aligned}
V_{\text {active }} & =V_{p} \frac{\tau_{2}}{\tau_{2}+\tau_{S L}} \rightarrow E_{\text {active }} \\
V_{S L} & =V_{p} \frac{\tau_{S L}}{\tau_{2}+\tau_{S L}} \rightarrow E_{S L}
\end{aligned}
$$

where $V_{p}$ is the applied voltage divided by the number of cascading stages.

However, one should be careful for the electric field calculation in the superlattice. In contrast to the Esaki-Tsu superlattice where the layer composition of quantum well and barrier is uniform so that a flat miniband condition is achieved even in the case of zero electric field [35], the design of superlattice in a QCL is nonuniform; an appropriate bias must apply before the Stark ladders are lined up and a flat miniband condition is met [18]. Therefore, the electric field for the electron transport is an effective electric field $E_{S L \text {,effective. In a simple picture, }}$ one can obtain $E_{S L \text {,effective }}$ by subtracting the flat band electric field $E_{S L \text {,flat }}$ from the applied electric field $E_{S L \text {,applied. This is }}$ schematically shown in Fig. 15.

In order to take into account the flat miniband condition in our serial circuit model, the voltage drop per stage for a flat band condition $V_{p \text {,flat }}$ was subtracted from the applied voltage drop per stage $V_{p}$. Therefore, (30) should read as

$$
V_{S L, \text { effective }}=\left(V_{p}-V_{p, \text { flat }}\right) \frac{\tau_{S L}}{\tau_{2}+\tau_{S L}} \rightarrow E_{S L, \text { effective }}
$$

where we obtained $V_{p \text {,flat }}$ by an independent bandstructure calculation to estimate the flat band condition for three differnet QCLs; the results are shown in Table II.

\section{B. Monte Carlo Simulation and Mobility}

The next issue is to estimate the spatial distribution of the equilibrium electron density throughout the superlattice at each bias condition. We have developed an ensemble Monte Carlo simulation to estimate the level-dependent electron density in the superlattice under steady-state conditions [36]. In the Monte Carlo simulation, we assume that the energy levels in one period of active and injector region consist of seven to ten energy levels within a typical miniband width of $\sim 150 \mathrm{meV}$ : the miniband width and energy levels differ depending on the different QCL designs, so it is calculated from an independent bandstructure 
TABLE II

EleCtric FIELd FOR a Flat MinibAND CONDITION AND LASING CONDITION

\begin{tabular}{c|c|c|c|c|c}
\hline Parameters & Electric field & $\mathbf{N 4 8 3}$ & $\mathbf{N 4 3 2}$ & $\mathbf{N 4 3 3}$ & Method \\
\hline Flat miniband condition & $E(\mathrm{kV} / \mathrm{cm})$ & 55 & 52 & 52 & Bandstructure calculation \\
\hline Lasing condition & $E(\mathrm{kV} / \mathrm{cm})$ & 73 & 96 & 94 & Measurement \\
\hline
\end{tabular}

TABLE III

PARAMETERS FOR DIELECTRIC RELAXATION

\begin{tabular}{c|c|c|c|c|c}
\hline Parameters & Description & $\mathbf{N 4 8 3}$ & $\mathbf{N 4 3 2}$ & $\mathbf{N 4 3 3}$ & Method \\
\hline Doping density & $10^{17}\left(\mathrm{~cm}^{3}\right)$ & 2.5 & 2.8 & 3.4 & Design parameter \\
\hline Number of cascading stages & $N_{p}$ & 22 & 25 & 25 & Design parameter \\
\hline Superlattice period & $d_{p}(\mathrm{~nm})$ & 5 & 5 & 5 & Design parameter \\
\hline Miniband width & $2 \Delta(\mathrm{meV})$ & 150 & 130 & 117 & Design parameter \\
\hline In-plane scattering & $\tau_{\|}(\mathrm{fs})$ & 42 & 42 & 42 & Assumed \\
\hline Calculated mobility & $\mu\left(\mathrm{cm}^{2} / \mathrm{Vs}\right)$ & 1818 & 1575 & 1418 & Calculated by Eq. 32 \\
\hline Effective mass & $m^{*}\left(m_{0}\right)$ & 0.04 & 0.04 & 0.05 & Calculated by Eq. 32 \\
\hline
\end{tabular}

simulation for the different QCL structures. Before starting the simulation, all electrons are assumed to be in the upper lasing state and the total number of electrons are assumed to be $2 \times 10^{4}$. In order to take into account the experimentally measured biasdependent lifetimes, the upper and lower state lifetimes from the rate-equation fits to the DT data are included in the simulation.

Each electron is labeled with its energy in the simulation. Electron dynamics is governed by: 1) electron-polar-optical-phonon scattering; 2) transport of electrons between stages; and 3) re-emission of electrons from the final state $f$ to the initial state $i$ due to the thermal effects where the Boltzmann factor $\exp \left(-\Delta E_{i f} / k_{b} T\right)$ was included. The electron population distribution at each time step is obtained through the sum of occupation of each energy level as well as the histogram of electron energy. Again, assuming no leakage current, electrons in final state of superlattice are re-pumped into the upper lasing state.

The resulting calculated electron density throughout the superlattice is about $1 \%-5 \%$ of the total electron density (doping density); the density in the upper part of the superlattice is the density relevant to estimating the dielectric relaxation time. The electron density profile differs from a Boltzmann-limited distribution since the bias-dependent scattering rates and lifetimes at each energy level make the density profile nonuniform across the superlattice.

In order to check the validity of our approach, we have calculated the superlattice mobility and the band-edge effective mass with the known QCL design parameters. Considering a 1-D model where the miniband conduction is in a low-field transport regime, the superlattice mobility is approximated as [37]

$$
\mu=\frac{q \Delta d_{p}^{2} \tau_{\|}}{\hbar^{2}}
$$

where $\hbar^{2} / \Delta d_{p}^{2}$ is the band-edge effective mass in the perpendicular transport in the superlattice and $\tau_{\|}$is the in-plane momentum scattering time. The relevant parameters for different QCL designs for the mobility and effective mass calculations are shown in Table III.

Using the parameters in Table III, the electron velocity in the superlattice was calculated by using the Esaki-Tsu velocityfield relation [35]

$$
v_{S L}=\frac{q \Delta d_{p}^{2} \tau_{\|}}{\hbar^{2}} \frac{E_{S L, \text { effective }}}{1+\left(q E_{S L, \text { effective }} d_{p} \tau_{\|} / \hbar\right)^{2}} .
$$

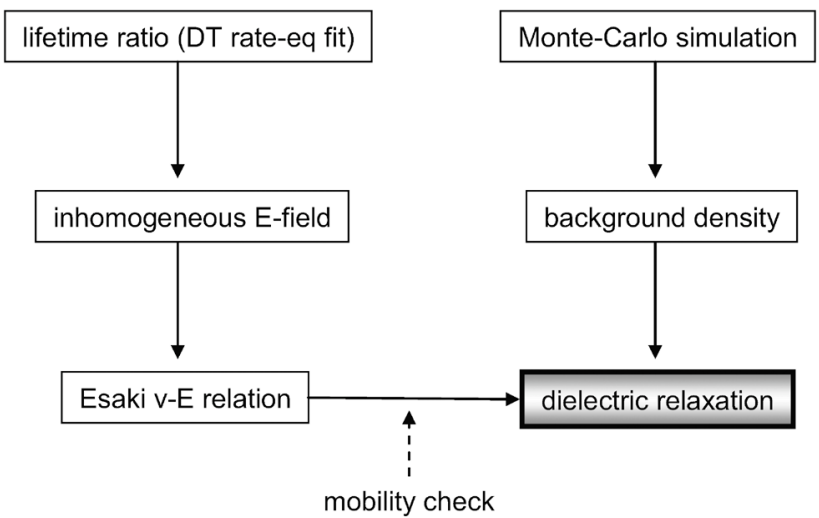

Fig. 16. Flow diagram to calculate the dielectric relaxation.

As shown in the next section, $\tau_{\|}(\simeq 42 \mathrm{fs})$ is the sole free parameter for the best fit to the Esaki-Tsu velocity-field relation; our estimation is also in good agreement with the previously reported in-plane momentum scattering time [38].

\section{Results}

The overall calculation procedures are summarized in Fig. 16 and the corresponding theoretical calculations of the dielectric relaxation time constants with the experimentally measured DT time constants are displayed in Figs. 17 and 18. Note that our approach of the simple serial circuit model and the Monte Carlo simulation have included the DT rate-equation fits and QCL design parameters, thus providing a self-consistent picture to understand the QCL superlattice dielectric relaxation; in-plane momentum scattering time is the only free parameter in our model. Furthermore, all DT data and calculations confirm that the superlattice transport is indeed in the mobility regime (a key assumption in the dielectric relaxation); note that the peak electric field in Figs. 17 and 18(a) is given with a good approximation by $E \simeq \hbar / q d_{p} \tau_{\|}$[37], which results in $\sim 30 \mathrm{kV} / \mathrm{cm}$, as shown in the figures.

In addition to N432 and N433 samples, we tested another sample (N483 QCL), which has a qualitatively different QCL design, being based on a four-QW active region with a slightly wider superlattice miniband width. More details on the gain recovery dynamics on the N483 QCL can be found elsewhere [39]; the key result is that the superlattice transport component of the gain recovery is found to follow the same dielectric relaxation model. 

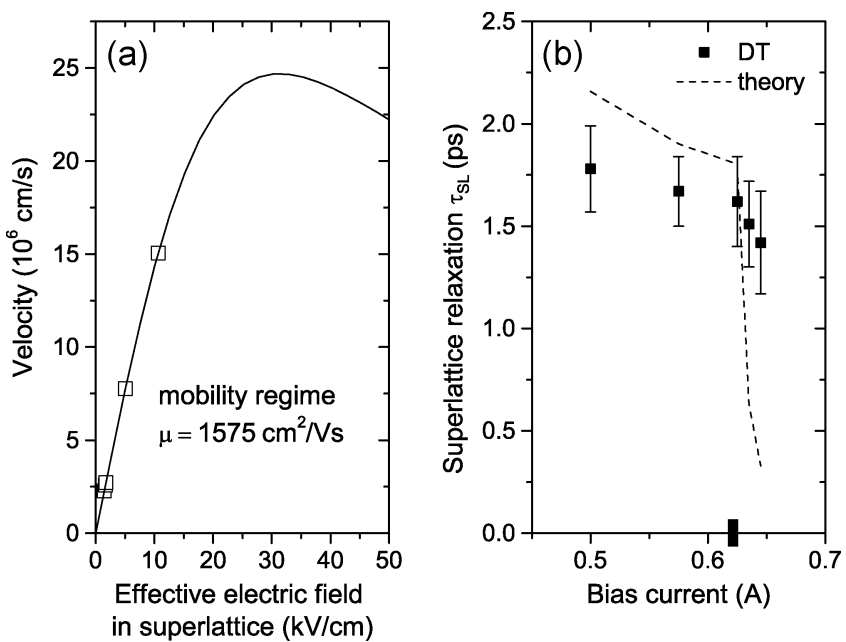

Fig. 17. Superlattice dielectric relaxation for the N432 QCL. (a) Velocity calculation based on the Esaki-Tsu model shows that the superlattice transport is in a linear regime. (b) DT rate-equation fits (squares) and the theoretical calculation of the dielectric relaxation (dashed line) are displayed. Threshold is indicated by the tick mark.
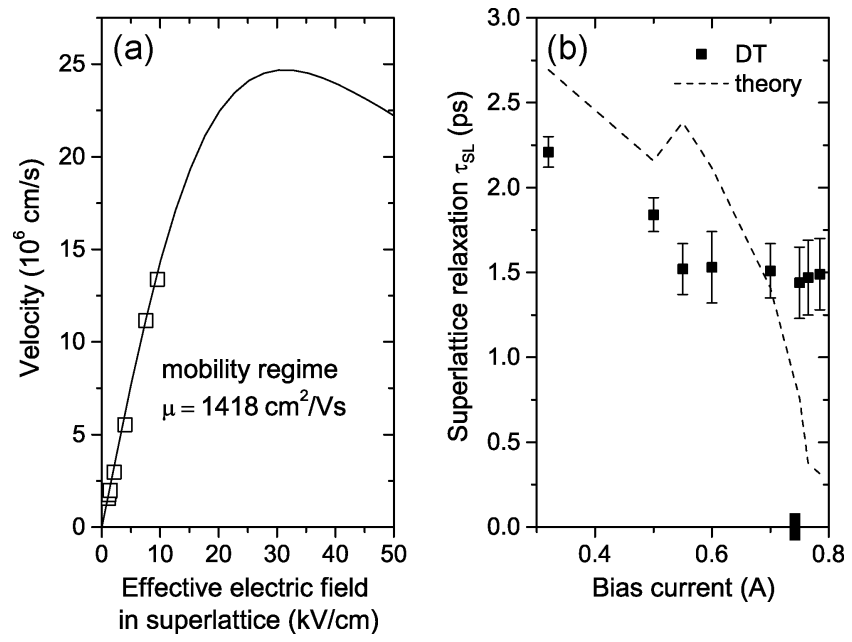

Fig. 18. Superlattice dielectric relaxation for the N433 QCL. (a) Velocity calculation based on the Esaki-Tsu model shows that the superlattice transport is in a linear regime. (b) DT rate-equation fits (squares) and the theoretical calculation of the dielectric relaxation (dashed line) are displayed. Threshold is indicated by the tick mark.

Fig. 17 shows data for the N432 QCL (this QCL has no intracavity saturable absorber in it). Data show a good agreement between the calculated dielectric relaxation and the measured $\tau_{S L}$; the structure of the N432 QCL is relatively simple, i.e., there is no resonant tunneling injection to achieve the population inversion or intra-cavity saturable absorber.

In Fig. 18, however, a significant deviation between the dielectric relaxation model and measured value for the N433 QCL is observed; N433 QCL structure has an intracavity saturable absorber (see the bandstructure in Fig. 1(b)). The discrepancies result from: 1) there might be a nonuniform voltage drop across the superlattice if, for example, the lifetime of energy levels in the saturable absorber QW is longer than the scattering rate, then this will result in complex field distributions near the saturable absorber region, and from there we overestimated the effective electric field compared to the case of uniform voltage drop and 2) the overestimated electric field indicates that the computed
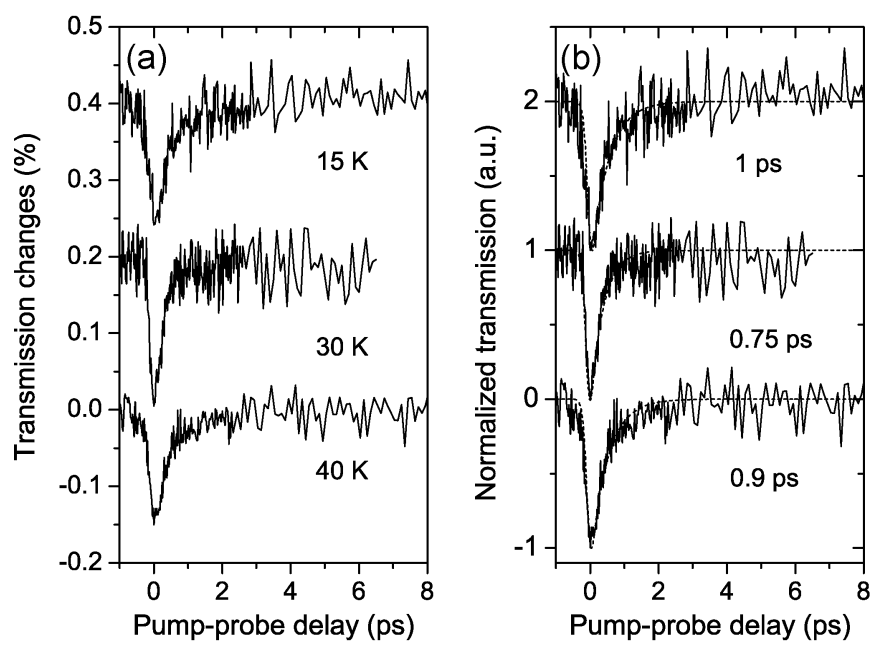

Fig. 19. (a) TE-pump and TM-probe DT measurement at $0.7 \mathrm{~A}$ for N433. The data offset for clarity. (b) Normalized transmission change (solid line) along with a fit (dashed line) with a single exponential decaying function convolved with the experimentally measured mid-IR pulse. All recovery time constants at various temperatures are $\sim 1 \mathrm{ps}$.

dielectric relaxation is underestimated in such a way that the calculated value is lower than the measured one.

\section{EXPERIMENTAL ARTIFACTS}

The interpretations of the DT dynamics given in the previous sections are made entirely in terms of the transport and relaxation processes in the cascade heterostructure. These interpretations require that possible sources of DT signals other than the population inversion dynamics must be small. Here, we consider two possible contributions to the DT signals.

\section{A. Hot Carrier Effects in the Upper Subband}

The polarizations of the pump and probe beams in our experiment are oriented at $\pm 45^{\circ}$ with respect to the transverse magnetic (TM) lasing mode of the QCL. This geometry enables both pump and probe beams to interact with the gain transition via the TM component, while the probe can be separated from the pump before detection by a polarizing analyzer. In order to isolate the effects of pump-induced carrier heating on the gain, we rotate the polarizations to transverse electric (TE)-pump and TM-probe. A TE-polarized pump only induces carrier heating [40]-[43] (via free-carrier or two-photon absorption) as it does not induce stimulated transitions from the upper lasing state. Fig. 19 shows the results of TE-pump/TM-probe DT measurements, indicating that the contribution of carrier heating to DT signals in the $45^{\circ}$ polarization is at most about $10 \%$ that of the stimulated emission contribution.

The observed $\sim 1$-ps recovery time constant is very similar to the cooling dynamics of the generated hot carriers, which has been previously observed in the interband laser diodes [40]-[43]. This process is essentially due to the effects of the hot electron generation, where a strong pump pulse may heat up the carrier by redistributing the electrons in the high energy state of the conduction band via two-photon absorption at high pump energy or free-carrier absorption at moderate pump energy. 


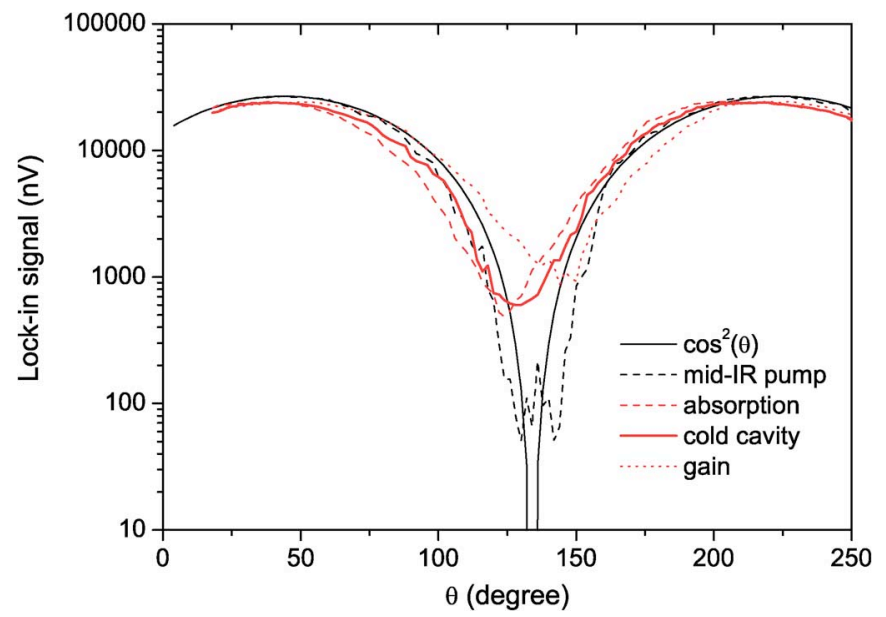

Fig. 20. Waveguide anisotropy and polarization rotation of mid-IR pulse. Amplitude changes of the transmitted mid-IR pulse through QC waveguide are shown as a function of polarizer angle $\theta$ when QCL at low bias (absorption, red dashed line), at high bias (gain, red dotted line), and at zero bias (cold cavity, red and thick solid line). For reference, calculated (black solid line) and measured (black dashed line) polarization rotation without QCL are also plotted.

\section{B. Waveguide Anisotropy}

The polarization anisotropy of the QCL waveguide may be another possible contributor to the measured gain dynamics. We checked the possible effect of waveguide anisotropy on the DT signals by measuring the rotation and ellipticity induced on the polarization as mid-IR pulses propagate through the QC structures. The transmitted pulse is analyzed using a linear polarizer placed in front of the $10-\mu \mathrm{m}$ spatial filter before the detector, and we plot the amplitude of the transmitted beam as a function of polarizer rotation angle $\theta$. For a reference, we also checked the polarization rotation in the QCL but without current biasing (cold cavity). The results are displayed in Fig. 20 and show only a few percent of the pump pulse leaks through the probe analyzer, and only a slight change of polarization is observed. Hence, the contribution of polarization anisotropy to the DT signals is believed to be small. We note, however, that if there is a nonlinear refraction contribution to the DT signal, both the gain and refraction nonlinearities arise from the carrier population dynamics; hence our conclusions regarding the carrier dynamics will be unchanged.

\section{CONCLUSION}

Our time-resolved measurements of the gain recovery dynamics in cascade heterostructure lasers show that the transport through the device and the cavity photon density are intimately coupled, and that from just below to above threshold, the current through the device is determined by the stimulated emission rate into the lasing mode. We note that analogous tunnelling of electrons via a photon-assisted transition has been previously observed in resonant tunnelling devices under the application of an external oscillating electric field [44]-[49]. In those experiments, the presence of a strong external classical field has opened up a new channel, increasing the transport across the device. In contrast to these experiments, we have shown that in
QCLs the current near and above threshold is driven by the intracavity photon density, and the effect of quantum stimulated emission on transport at the few-photon level can be seen to turn on as the laser approaches threshold from below. Dynamics of the lower state emptying has been investigated by using tightbinding tunneling model and phonon-limited scattering. Superlattice transport has been extensively studied using a simple dielectric relaxation model with bias-dependent inhomogeneous electron field distributions. Possible other contributions to the DT, such as carrier heating effect and waveguide anisotropy, are separately investigated through the polarization-dependent pump-probe measurements and shown to be small.

\section{ACKNOWLEDGMENT}

The authors would like to thank A. Belyanin for fruitful discussion and critical reading.

\section{REFERENCES}

[1] J. Faist, F. Capasso, D. L. Svico, C. Sirtori, A. L. Hutchinson, and A. Y. Cho, "Quantum cascade lasers," Science, vol. 264, pp. 553-556, 1994.

[2] M. Beck, D. Hofstetter, T. Aellen, J. Faist, U. Oesterle, M. Ilegems, E. Gini, and H. Melchior, "Continuous wave operation of a midinfrared semiconductor laser at room temperature," Science, vol. 79, pp. 301-305, 2002.

[3] A. Evans, S. R. Darvish, S. Slivken, J. Nguyen, Y. Bai, and M Razeghia, "Buried heterostructure quantum cascade lasers with high continuous-wave wall plug efficiency," Appl. Phys. Lett., vol. 91, pp. $0711011-0711013,2007$.

[4] L. Diehl, D. Bour, S. Corzine, J. Zhu, G. Höfler, M. Lončar, M. Troccoli, and F. Capasso, "High-power continuous wave operation of strainbalanced quantum cascade lasers by metal organic vapor-phase epitaxy," Appl. Phys. Lett., vol. 89, pp. 081 101-1-081 101-3, 2006.

[5] R. Paiella, R. Martini, F. Capasso, C. Gmachl, H. Y. Hwang, D. L. Svico, J. N. Baillargeon, A. Y. Cho, E. A. Whittaker, and H. C. Liu, "High-frequency modulation without the relaxation oscillation resonance in quantum cascade lasers," Appl. Phys. Lett., vol. 79, pp. 2526-2528, 2001.

[6] R. Terazzi, T. Gresch, M. Giovannini, N. Hoyler, N. Sekine, and J. Faist, "Bloch gain in quantum cascade lasers," Nature Phys., vol. 3 , pp. 329-333, 2007.

[7] R. Köhler, A. Tredicucci, F. Beltram, H. E. Beere, E. H. Linfield, A. G. Davies, D. A. Ritcher, R. C. Iotti, and F. Rossi, "Teraherz semiconductor-heterostructure laser," Nature, vol. 417, pp. 156-159, 2002

[8] M. A. Belkin, F. Capasso, A. Belyanin, D. L. Sivco, A. Y. Cho, D. C. Oakley, C. J. Vineis, and G. W. Turner, "Terahertz quantum-cascade-laser source based on intracavity difference-frequency generation," Nature Photon., vol. 1, pp. 288-292, 2007.

[9] S. Lutgen, R. A. Kaindl, M. Woerner, T. Elsaesser, A. Hase, H. Kunzel, M. Gulia, D. Meglio, and P. Lugli, "Nonequilibrium dynamics in a quasi-two-dimensional electron plasma after ultrafast intersubband excitation," Phys. Rev. Lett., vol. 77, pp. 3657-3660, 1996.

[10] S. Lutgen, R. A. Kaindl, M. Woerner, T. Elsaesser, A. Hase, and H. Kunzel, "Nonlinear intersubband absoprtion of a hot quasi-two-dimensional electron plasma studied by femtosecond infrared spectroscopy," Phys. Rev. B, vol. 54, pp. 17 343-17 346, 1996.

[11] R. A. Kaindl, S. Lutgen, M. Woerner, T. Elsaesser, B. Nottelmann, V. M. Axt, T. Kuhn, A. Hase, and H. Künzel, "Ultrafast dephasing of coherent intersubband polarizations in a quasi-two-dimensional electron plasma," Phys. Rev. Lett., vol. 80, pp. 3575-3579, 2001.

[12] R. A. Kaindl, K. Reimann, M. Woerner, T. Elsaesser, R. Hey, and K. H. Ploog, "Homogeneous broadening and excitation-induced dephasing of intersubband transitions in a quasi-two-dimensional electron gas," Phys. Rev. B, vol. 63, pp. 1613 081-1 613 184, 2001.

[13] F. Eickemeyer, K. Reimann, M. Woerner, T. Elsaesser, S. Barbieri, C. Sirtori, G. Strasser, T. Muller, R. Bratschitsch, and K. Unterrainer, "U1trafast coherent electron transport in semiconductor quantum cascade structures," Phys. Rev. Lett., vol. 89, pp. 474 021-474 024, 2002.

[14] F. Eickemeyer, "Nonlinear optics and short pulse spectroscopy," Ph.D. dissertation, Max-Born Institute, , 2002. 
[15] R. C. Iotti and F. Rossi, "Nature of charge transport in quantum-cascade lasers," Phys. Rev. Lett., vol. 87, pp. 146 603-1-146 603-4, 2001.

[16] S.-C. Lee, F. Banit, M. Woerner, and A. Wacker, "Quantum mechanical wavepacket transport in quantum cascade laser structures," Phys. Rev. $B$, vol. 73, pp. 245 320-1-245 310-6, 2006.

[17] C. Weber, F. Banit, S. Butscher, A. Knorr, and A. Wacker, "Theory of the ultrafast nonlinear response of teraherz quantum cascade laser structures," Appl. Phys. Lett., vol. 89, pp. 091 112-1-091 112-3, 2006.

[18] J. Faist, F. Capasso, D. L. Svico, C. Sirtori, and A. Y. Cho, Quantum Cascade Lasers in Intersubband Transitions in Quantum Wells: Physics and Device Applications II. New York: Academic, 2000.

[19] J. Kröll, J. Darmo, S. S. Dhillon, X. Marcadet, M. Calligaro, C. Sirtori, and K. Unterrainer, "Phase-resolved measurements of stimulated emission in a laser," Nature, vol. 449, pp. 698-701, 2007.

[20] H. Choi, L. Diehl, Z.-K. Wu, M. Giovanni, J. Faist, F. Capasso, and T. B. Norris, "Gain recovery dynamics and photon-driven transport in quantum cascade lasers," Phys. Rev. Lett., vol. 100, pp. 167 401-1-167 401-4, 2008.

[21] J. Faist, F. Capasso, C. Sirtori, D. L. Svico, A. L. Hutchinson, and A. Y. Cho, "Laser action by tuning the oscillator strength," Nature, vol. 387, pp. 777-782, 1997.

[22] C. Y. Wang, L. Diehl, A. Gordon, C. Jirauschek, F. X. Kärtner, A. Belyanin, D. Bour, S. Corzine, G. Hofler, M. Troccoli, J. Faist, and F. Capasso, "Coherent instabilities in a semiconductor laser with fast gain recovery," Phys. Rev. A, vol. 75, pp. 031 802-1-031 802-4, 2007.

[23] A. E. Siegman, Lasers. Sausalito, CA: University Science, 1986.

[24] D. Y. Oberli, J. Shah, T. C. Damen, C. W. Tu, T. Y. Chang, D. A. B. Miller, J. E. Henry, R. F. Kopf, N. Sauer, and A. E. DiGiovanni, "Direct measurement of resonant and nonresonant tunneling times in asymmetric coupled quantum wells," Phys. Rev. B, vol. 40, pp. 3028-3031, 1989.

[25] S. Blaser, L. Diehl, M. Beck, J. Faist, U. Oesterle, J. Xu, S. Barbieri, and F. Beltram, "Characterization and modeling of quantum cascade lasers based on a photon-assisted tunneling transition," IEEE J. Quantum Electron., vol. 37, no. 3, pp. 448-455, Mar. 2001.

[26] R. Ferreira and G. Bastard, "Evaluation of some scattering times for electrons in unbiased and biased single- and multiple-quantum-well structures," Phys. Rev. B, vol. 40, pp. 1074-1086, 1989.

[27] M. Lundstrom, Fundamentals of Carrier Transport. Cambridge, U.K.: Cambridge Univ. Press, 2000.

[28] A. Yariv, Quantum Electronics. New York: Wiley, 1989.

[29] P. W. Milonni and J. H. Eberly, Lasers. New York: Wiley Interscience, 1988.

[30] K. Leo, J. Shah, J. P. Gordon, T. C. Damen, D. A. B. Miller, C. W. $\mathrm{Tu}$, and J. E. Cunningham, "Effect of collisions and relaxation on coherent resonant tunneling: Hole tunneling in $\mathrm{GaAs} / \mathrm{Al}_{x} \mathrm{Ga}_{1-x} \mathrm{As}$ double-quantum-well structures," Phys. Rev. B, vol. 42, pp. 7065-7068, 1990.

[31] B. Williams, "Terahertz quantum cascade lasers," Ph.D. dissertation, Dept. of Elect. Eng. and Comput. Sci., MIT, Cambridge, 2003.

[32] J. Singh, Physics of Semiconductor and Their Heterostructures. New York: McGraw-Hill, 1993.

[33] M. Woerner, K. Reimann, and T. Elsaesser, "Coherent charge transport in semiconductor quantum cascade structures," J. Phys.: Condens. Matter, vol. 14, pp. R25-R48, 2004.

[34] S. M. Sze, Physics of Semiconductor Devices. New York: Wiley, 1981.

[35] L. Esaki and R. Tsu, "Superlattice and negative differential conductivity in semiconductors," IBM J. Res. Develop., vol. 89, pp. 61-65, 1970.

[36] Z.-K. Wu, H. Choi, X. Su, S. Chakrabarti, P. Bhattacharya, and T. B. Norris, "Ultrafast electronic dynamics in unipolar n-doped InGaAs/ GaAs self-assembled quantum dots," IEEE J. Quantum Electron., vol. 43, no. 4, pp. 486-496, Apr. 2007.

[37] F. Capasso, K. Mohammed, and A. Y. Choi, "Resonant tunneling through double barriers, perpendicular quantum transport phenomena in superlattices, and their device applications," IEEE J. Sel. Top. Quantum Electron., vol. QE-22, no. 6, pp. 1853-1869, Nov./Dec. 1986.

[38] C. Sirtori, F. Capasso, J. Faist, A. Hutchinson, D. L. Sivco, and A. Y. Cho, "Resonant tunneling in quantum cascade lasers," IEEE $J$. Quantum Electron., vol. 34, no. 9, pp. 1722-1729, Sep. 1998.

[39] H. Choi, T. B. Norris, T. Gresch, M. Giovanni, J. Faist, L. Diehl, and F. Capasso, "Femtosecond dynamics of resonant tunneling and superlattice relaxation in quantum cascade lasers," Appl. Phys. Lett., vol. 92, pp. 122 114-1-122 114-3, 2008.
[40] K. L. Hall, G. Lenz, E. P. Ippen, U. Koren, and G. Raybon, “Carrier heating and spectral hole burning in strained-layer quantum-well laser amplifiers at $1.5 \mu \mathrm{m}$," Appl. Phys. Lett., vol. 61, pp. 2512-2514, Nov. 1992.

[41] C.-K. Sun, H. K. Choi, C. A. Wang, and J. G. Fujimoto, "Studies of carrier heating in InGaAs/AlGaAs strained-layer quantum well diode lasers using a multiple wavelength pump probe technique," Appl. Phys. Lett., vol. 62, pp. 747-749, 1993.

[42] C.-K. Sun, H. K. Choi, C. A. Wang, and J. G. Fujimoto, "Femtosecond gain dynamics InGaAs/AlGaAs strained-layer single quantum-well diode lasers," Appl. Phys. Lett., vol. 63, pp. 96-98, Jul. 1993.

[43] K. L. Hall, G. Lenz, A. M. Darwish, and E. P. Ippen, "Subpicosecond gain and index nonlinearities in InGaP diode lasers," Opt. Commun., vol. 111, pp. 589-612, 1994.

[44] A. H. Dayem and R. J. Martin, "Quantum interaction of microwave radiation with tunneling between superconductors," Phys. Rev. Lett., vol. 8, pp. 246-248, 1962.

[45] P. K. Tien and J. P. Gordon, "Multiphoton process observed in the interaction of microwave fields with the tunneling between superconductor films," Phys. Rev., vol. 129, pp. 647-651, 1963.

[46] P. S. S. Guimaraes, B. J. Keay, J. P. Kaminski, J. S. J. Allen, P. F. Hopkins, A. C. Gossard, L. T. Florez, and J. P. Harbison, "Photon-mediated sequential resonant tunneling in intense terahertz electric fields," Phys. Rev. Lett., vol. 70, pp. 3792-3795, 1993.

[47] B. J. Keay, S. Zeuner, J. S. J. Allen, K. D. Maranowski, A. C. Gossard, U. Bhattacharya, and M. J. W. Rodwell, "Dynamic localization, absolute negative conductance, and stimulated, multiphoton emission in sequential resonant tunneling semiconductor superlattices," Phys. Rev. Lett., vol. 75, pp. 4102-4105, 1995.

[48] B. J. Keay, J. S. J. Allen, J. Galán, J. P. Kaminski, K. L. Campman, A. C. Gossard, U. Bhattacharya, and M. J. W. Rodwell, "Photon-assisted electric field domains and multiphoton-assisted tunneling in semiconductor superlattices," Phys. Rev. Lett., vol. 75, pp. 4098-4101, 1995.

[49] K. Unterrainer, B. J. Keay, M. C. Wanke, J. S. J. Allen, D. Leonard, G. Medeiros-Ribeiro, U. Bhattacharya, and M. J. Rodwell, "Inverse bloch oscillator: Strong terahertz-photocurrent resonances at the bloch frequency," Phys. Rev. Lett., vol. 76, pp. 2973-2975, 1996.

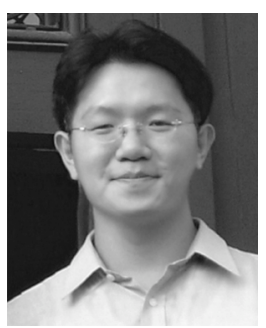

Hyunyong Choi (M'06) received the B.S. degree in electrical and electronic engineering from Yonsei University, Seoul, Korea, in 2002 and the M.S. and $\mathrm{Ph} . \mathrm{D}$. degrees in electrical engineering and computer science from the University of Michigan, Ann Arbor, in 2004 and 2007, respectively. For his Ph.D. thesis, he investigated ultrafast electronic transport dynamics in quantum cascade lasers.

Since then, he has been a Postdoctoral Fellow in Materials Sciences Division, Lawrence Berkeley National Laboratory and Department of Physics, University of California, Berkeley, where he has been working on the application of terahertz spectroscopy to semiconductor nanostructures.

Dr. Choi is a member of the American Physical Society and the Optical Society of America.

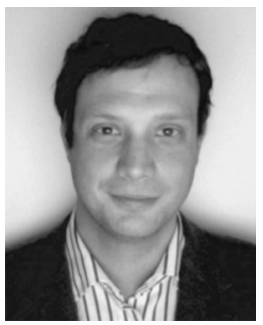

Laurent Diehl was born in Switzerland in 1976. $\mathrm{He}$ received the Ph.D. degree from the University of Neuchâtel, Neuchâtel, Switzerland, in 2003. His work concentrated on the development of p-type $\mathrm{Si} / \mathrm{SiGe}$ quantum cascade lasers.

He joined the group of Prof. F. Capasso at Harvard University, Cambridge, in 2003 as a Postdoctoral Fellow and later as a Research Associate. His work currently focuses on the development of highpower quantum cascade lasers grown by MOCVD, as well as the physics of these devices, including their ultrafast dynamics. 2001. 


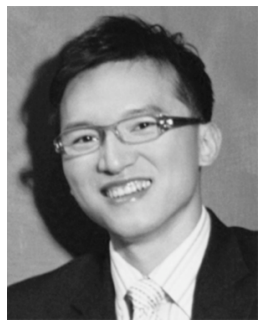

Zong-Kwei Wu received the B.S. degree from the National Taiwan University, Taipei, Taiwan, in 1998 and the M.S. and Ph.D. degrees from the University of Michigan, Ann Arbor, in 2002 and 2006, respectively, all in electrical engineering and computer science.

He was a Postdoctoral Research Fellow with the Center for Ultrafast Optical Science, University of Michigan, in 2006. His research interests include ultrafast dynamics in semiconductor nanostructures. He joined Intel Corporation, Santa Clara, CA, in developing optical metrology on semiconductor thin films and structures.

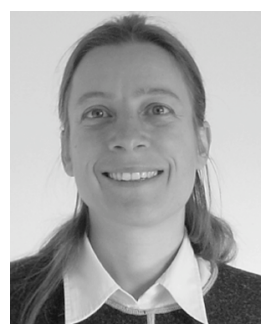

Marcella Giovannini was born in 1970. She received the $\mathrm{Ph}$.D. degree in physics from the ETH, Lausanne, Switzerland. Her studies focused on low-dimensional properties of metallic structures.

After a first postdoctoral position on photoemission studies of mono-atomic metallic chains, in 2001 she joined the Mesoscopic group led by Prof. J. Faist at the Institute of Physics, University of Neuchâtel, Neuchâtel, Switzerland, where her work was dedicated to the study of InP- and GaAs-based mid and far-infrared QC lasers. Since 2007, she has been with the Institute of Microtechnology, University of Neuchâtel.

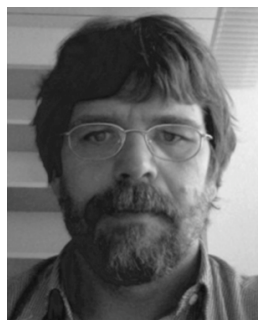

Jérôme Faist (M’08) was born in Geneva, Switzerland. He received the B.S. and Ph.D. degrees in physics from the Swiss Institute of Technology (ETH), Lausanne, in 1985 and 1989 respectively.

After a postdoctoral position with IBM Rueschlikon (1989-1991), he joined F. Capasso's group at Bell Laboratories in 1991, where he worked first as a Postdoctoral Researcher and then as a Member of Technical Staff. From 1997 to 2007, he was a Professor with the Physics Institute, University of Neuchâtel, Neuchâtel, Switzerland. In 2007, he became a Professor with the Institute for Quantum Electronics, ETH Zurich.

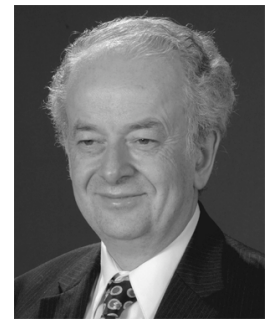

Federico Capasso (F'87) received the Ph.D. degree in physics (summa cum laude) from the University of Rome, Rome, Italy, in 1973.

$\mathrm{He}$ is the Robert Wallace Professor of Applied Physics and Vinton Hayes Senior Research Fellow in Electrical Engineering at Harvard University, Cambridge, MA. He then joined Fondazione Ugo Bordoni as a Researcher from 1974 to 1976 . He joined Bell Laboratories in 1976, where he held positions as a Member of Technical Staff (1977-1986), Department Head (1986-2000), and Vice President for Physical Research (2000-2002). In 1997, he was made a Bell Labs Fellow for his scientific contributions. He has been engaged in basic and applied research on the design of new artificial materials and devices, using an approach he pioneered known as band structure engineering, in investigations on quantum electrodynamical phenomena such as the Casimir effect and on surface plasmon photonics. This research has opened up new directions in electronics, photonics, mesoscopic physics and nanoscale science and technology. $\mathrm{He}$ is a coinventor of the quantum cascade laser, a fundamentally new light source, which has now been commercialized. His recent achievements include the invention and demonstration of low divergence semiconductor lasers with plasmonic collimators, plasmonic laser antennas and the first measurement of repulsive Casimir forces. He has coauthored over 300 papers, edited four volumes, and holds over 55 U.S. patents.

Dr. Capasso is a Fellow of the American Academy of Arts and Sciences, the Optical Society of America, the American Physical Society, the International Society for Optical Engineering (SPIE), and the American Association for the Advancement of Science (AAAS), a member of the National Academy of Sciences and the National Academy of Engineering, and an honorary member of the Franklin Institute. His awards include the King Faisal International Prize for Science, the IEEE Edison Medal, the Arthur Schawlow Prize in Laser Science, the Wetherill Medal of the Franklin Institute, the Robert Wood Prize of the Optical Society of America (OSA), the William Streifer Award of the Laser and Electro-optic Society (IEEE), the Rank Prize in Optoelectronics (UK), the IEEE David Sarnoff Award in Electronics, the Duddell Medal of the Institute of Physics (UK), the Willis Lamb Medal for Laser Science and Quantum Optics, the Materials Research Society Medal, the "Vinci of Excellence" Prize (France), the Welker Memorial Medal (Germany), the New York Academy of Sciences Award, the Newcomb Cleveland Prize of the American Association for the Advancement of Science.

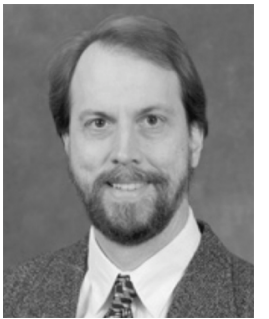

Theodore B. Norris (A'95) received the B.A. degree (with Highest Honors) from Oberlin College, Oberlin, $\mathrm{OH}$, in 1982 and the Ph.D. degree from the University of Rochester, Rochester, NY, in 1989 , both in physics. His postdoctoral research was performed at Thomson-CSF in France during 1989-1990

$\mathrm{He}$ is a Professor with the Electrical Engineering and Computer Science Department and Director of the Center for Ultrafast Optical Science at the University of Michigan, Ann Arbor. His research interests include application of femtosecond optical techniques to the physics of semiconductor nanostructures, in developing new ultrafast optical and optoelectronic measurement techniques, $\mathrm{THz}$ generation and measurement, plasmonics in nanostructues, and novel methods for biological imaging and in vivo sensing.

Dr. Norris is a Fellow of the Optical Society of America and the American Physical Society. 